\title{
The Interaction of Supernova Reminants with Interstellar Clouds: Experiments on the Nova Laser
}

R.L. Klein, K.S. Budil, T.S. Perry, D.R. Bach

April 18, 2002

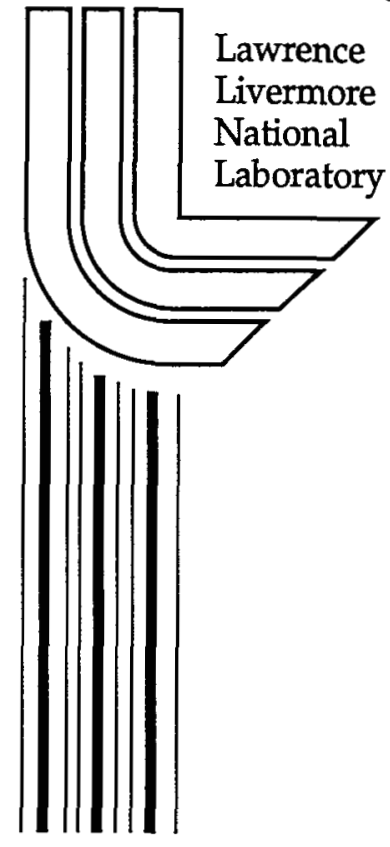




\section{DISCLAIMER}

This document was prepared as an account of work sponsored by an agency of the United States Government. Neither the United States Government nor the University of California nor any of their employees, makes any warranty, express or implied, or assumes any legal liability or responsibility for the accuracy, completeness, or usefulness of any information, apparatus, product, or process disclosed, or represents that its use would not infringe privately owned rights. Reference herein to any specific commercial product, process, or service by trade name, trademark, manufacturer, or otherwise, does not necessarily constitute or imply its endorsement, recommendation, or favoring by the United States Government or the University of California. The views and opinions of authors expressed herein do not necessarily state or reflect those of the United States Government or the University of California, and shall not be used for advertising or product endorsement purposes.

This work was performed under the auspices of the U.S. Department of Energy by the University of California, Lawrence Livermore National Laboratory under Contract No. W-7405-Eng-48.

This report has been reproduced directly from the best available copy.

Available electronically at http://www.doc.gov/bridge

Available for a processing fee to U.S. Department of Energy

And its contractors in paper from

U.S. Department of Energy

Office of Scientific and Technical Information

P.O. Box 62

Oak Ridge, TN 37831-0062

Telephone: (865) 576-8401

Facsimile: (865) 576-5728

E-mail: reports@adonis.osti.gov

Available for the sale to the public from

U.S. Department of Commerce

National Technical Information Service

5285 Port Royal Road

Springfield, VA 22161

Telephone: (800) 553-6847

Facsimile: (703) 605-6900

E-mail: orders@ntis.fedworld.gov

Online ordering: http://www.ntis.gov/ordering.htm

OR

Lawrence Livermore National Laboratory

Technical Information Department's Digital Library

http://www.llnl.gov/tid/Library.html 


\title{
THE INTERACTION OF SUPERNOVA REMINANTS WITH INTERSTELLAR CLOUDS: EXPERIMENTS ON THE NOVA LASER
}

(Short Title: SHOCK-CLOUD INTERACTION LASER EXPERIMENTS)

Richard I. Klein ${ }^{1,2}$, Kimberly S. Budil ${ }^{2}$, Theodore S. Perry ${ }^{2}$, and David R. Bach ${ }^{2}$

${ }^{1}$ University of California, Berkeley, Dept. of Astronomy, 601 Campbell Hall, Berkeley, California 94720; klein@astron.berkeley.edu

${ }^{2}$ Lawrence Livermore National Laboratory, P. O. Box 808, Livermore, California 94550; budi11@1lnl.gov, perry15@1lnl.gov, bach3@lln1.gov

\begin{abstract}
The interaction of strong shock waves, such as those generated by the explosion of supernovae with interstellar clouds, is a problem of fundamental importance in understanding the evolution and the dynamics of the interstellar medium (ISM) as it is disrupted by shock waves. Here we present the results of a series of Nova laser experiments investigating the evolution of a high density sphere embedded in a low density medium after the passage of a strong shock wave, thereby emulating the supernova shock-cloud interaction. The Nova laser was utilized to generate a strong ( Mach 10$)$ shock wave that traveled along a miniature beryllium shock tube, $750 \mu \mathrm{m}$ in diameter, filled with a low-density plastic emulating the ISM. Embedded in the plastic was a copper microsphere (100 $\mu \mathrm{m}$ in diameter) emulating the interstellar cloud. The morphology and evolution as well as the shock wave trajectory were diagnosed via side-on $\mathrm{X}$-ray radiography.
\end{abstract}

This work was performed under the auspices of the U.S. Department of Energy by the University of California, Lawrence Livermore National Laboratory under contract No. W-7405-Eng-48. 
We describe here experimental results of this interaction out to several cloud crushing times and compare them to detailed two and three dimensional radiation hydrodynamic simulations using both arbitrary Lagrangian and Eulerian hydrodynamics (ALE) as well as high resolution AMR hydrodynamics.

Subject headings: methods: laboratory - ISM: clouds - supernova remnants - shock waves

\section{INTRODUCTION}

The interaction of shock waves in the interstellar medium (ISM), such as those as those associated with supernovae, stellar winds, bipolar flows, HII regions or spiral density waves with interstellar clouds is a fundamental problem in interstellar gas dynamics and is crucial to understanding the evolution of the ISM. The physics of this essential interaction sheds light on several key questions: (1) What is the rate and total amount of gas stripped from the cloud, and what are the mechanisms responsible? (2) What is the rate of momentum transfer to the cloud? (3) What is the appearance of the shocked cloud, its morphology and velocity dispersion? (4) What is the role of vortex dynamics on the evolution of the cloud? (5) Can the interaction result in the formation of a new generation of stars? The investigation of this highly non-linear interaction is not amenable to analytic approaches and requires a detailed multi-dimensional hydrodynamic study of the shock-cloud problem using high-resolution numerical methods. Such an approach has been made by Klein, McKee and Colella (1994), henceforth (KMC) who found that the cloud may be destroyed by a series of instabilities associated with the post-shock flow of intercloud gas past the cloud. Earlier work on this important problem includes that of Woodward (1976) and Nittman, Falle, \& Gaskell (1982). More recent work that investigates the deposition of vorticity associated with the shock bubble interaction and the evolution of vortex structures can be found (Zabusky, Zeng, Ray, \&. Samteney 1997). Recently, Levinson et al. (1997) performed a detailed x-ray survey of 
the Cygnus Loop supernova remnant with the ROSAT High Resolution Imager. The Cygnus Loop is the prototype for a supernova remnant (SNR) that is dominated by ISM-shock interactions. The Cygnus Loop provides key observations showing the basic inhomogeneity of the ISM and the fundamental nature of shock-cloud interactions and their role in determining the $\mathrm{x}$-ray morphology of the supernova remnant.

A question of key importance is whether one can assess the validity of the results of the complex multi-dimensional calculations necessary to understand the morphological evolution of the shocked interstellar cloud and its associated instabilities. It has recently been shown (Ryutov et al. 1999) that under certain conditions, the Euler equations of hydrodynamics, whose numerical solution provides a deep understanding of this fundamental fluid interaction, are self-similarly invariant to certain scale transformations. This scaling opens the door to understanding this fundamental interaction, which occurs on galactic scales, by performing well characterized laboratory experiments, which occur on scales that are some 20 orders of magnitude smaller in size. In this paper, we describe a set of new experiments, performed on the Nova high energy density laser at Lawrence Livermore National Laboratory to investigate this fundamental fluid dynamical shock sphere interaction and to shed light on recent astronomical observations. These experiments serve a dual purpose. They allow us to validate against laboratory data, the complex hydrodynamic code simulations that we use to investigate the interstellar blast wave interaction with atomic or molecular clouds. In addition, they provide us with a controlled laboratory environment with which to investigate in detail aspects of the key instabilities that we believe are paramount in determining the fate of interstellar clouds. Because these laboratory experiments scale selfsimilarly to the astrophysical phenomena, we have a powerful new approach to studying the interstellar laboratory and deriving diagnostic information that would otherwise be impossible to obtain through astronomical observations. 
We compare X-ray radiographs obtained from the Nova laser experiments to detailed 2D multi-fluid radiation-hydrodynamic calculations of the experiment carried out with a multifluid, arbitrary Lagrangian-Eulerian code (CALE) (Kane et al. 1997) and we discuss key features of the interaction such as the drag velocity of the shocked cloud, the destruction time of the cloud, the morphological evolution of the cloud, and the break up of the cloud by hydrodynamic instabilities. We address the limitations of these 2D calculations to explain the development of three-dimensional instabilities that are evident in the experimental radiographs at late times when instabilities result in breaking the initial symmetry of the cloud. Scaling the results from the shocked microspheres on the NOVA laser to the scales of interstellar clouds are discussed and applications to observations of the Cygnus Loop are made.

In section 2 we formulate the relevant timescales in the shock-cloud interaction and show how these timescales are related to hydrodynamic instabilities that govern the fate of the cloud. In section 3 we describe the experimental setup for investigating shock-sphere interactions on a high energy density facility such as the Nova laser. In section 4 we discuss properties of the equations of hydrodynamics that permit scaling from characteristic length scales of the laser experiments, 10 's of microns, up 20 orders of magnitude in scale to atomic and molecular clouds in galactic SNR that have characteristic length scales on the order of a parsec. In section 5 we discuss $2 \mathrm{D}$ radiation-hydrodynamic simulations of the experiment and their associated simulated radiographs. Full 3D simulations of the shock-cloud interaction and comparison with experiments is deferred to a later paper. In section 6 we discuss comparison of the simulated radiographs from the calculations with experimental radiographs obtained with X-ray shadowgraphy from the laser experiment and in section 7 we show how these results relate to recent observations of shock-cloud interactions in the Cygnus Loop SNR. In conclusion, we discuss new directions in on going experiments. The 
results of the present paper are a detailed explication of the recent work presented by Klein et al. (2000).

\section{RELEVANT TIME SCALES}

To describe the evolution of a shocked cloud as applied to the interstellar medium as well as the evolution of shocked sphere in the Nova laser experiments we follow the formulation of the earlier work of (KMC). We consider a cloud in pressure equilibrium with an ambient medium density of $\rho_{i 0}$ (Fig.1). We consider the case of a spherical cloud with radius $\mathrm{a}_{0}$. If the cloud is approximately isothermal and gravity and magnetic fields are negligible, the cloud will have an essentially uniform density $\rho_{c 0}$. We then define the density contrast between the cloud and the surrounding inter-cloud medium (ICM),

$$
\chi \equiv \frac{\rho_{c 0}}{\rho_{i 0}}
$$

This is expected to be of order $10^{2}$ for cold atomic clouds $\left(\mathrm{T} \sim 10^{2}{ }^{\circ} \mathrm{K}\right)$ embedded in either the warm neutral medium or the photo-ionized warm medium $\left(\mathrm{T} \sim 10^{4}{ }^{\circ} \mathrm{K}\right)$; a similar density contrast is expected for a cloud of warm gas embedded in the coronal gas $\left(\mathrm{T} \sim 10^{6}{ }^{\circ} \mathrm{K}\right)$. Higher contrasts are possible for molecular clouds $\left(\mathrm{T} \sim 10^{\circ} \mathrm{K}\right)$ embedded in warm gas $\left(\sim 10^{3}\right)$ or cold atomic clouds embedded in coronal gas $\left(\sim 10^{4}\right)$.

When a shock wave that is propagating through the inter-cloud medium at velocity $v_{b}$ encounters a cloud, it drives a shock into the cloud. Although the velocity of the shock changes as it moves through the cloud, its typical value $v_{s}$ is given by pressure balance with the shocked inter-cloud medium. If the initial shockwave is strong $(M \gg>1)$, pressure balance gives

$$
v_{s} \cong\left(\frac{\rho_{i 0}}{\rho_{c 0}}\right)^{1 / 2} v_{b}, \quad v_{s}=v_{b} \chi^{-1 / 2}
$$


This assumes that the shock in the ICM is not radiative. The time for the initial shock in the ICM to traverse the cloud is

$$
t_{i c} \equiv \frac{2 a_{0}}{v_{b}}
$$

The time for the cloud to be crushed by the shocks moving into the cloud is $a_{0} / v_{s}$. Using equation (2.2) we can define the cloud crushing time as

$$
t_{c c}=\frac{\chi^{1 / 2} a_{0}}{v_{b}}
$$

This is the characteristic timescale that governs the evolution of the shocked cloud.

McKee et al. (1978) showed that the blast wave of a supernova remnant (SNR) can accelerate the cloud in two stages. The shock-cloud first accelerates it to a velocity $v_{s}$, and the flow of shocked inter-cloud gas then accelerates it until it is co-moving with the shocked inter-cloud gas, which has a velocity $\frac{3}{4} v_{b}$ if one assumes an ideal gas with $\gamma=\frac{5}{3}$. As shown by KMC, the characteristic cloud drag time for a strong shock becomes

$$
t_{d r a g, 0}=\frac{\chi a_{0}}{c_{d} v_{b}}=\frac{\chi^{1 / 2} t_{c c}}{c_{d}}
$$

under the assumption that the cross-sectional area of the cloud remains constant during the cloud's acceleration and the drag coefficient is $c_{d}$. In practice, the cloud undergoes substantial lateral expansion and the drag time turns out to be a few times $t_{c c}$, considerably smaller than equation (2.5). After the initial shock has swept over the cloud, the cloud undergoes both Kelvin-Helmholtz and Rayleigh Taylor instabilities. For $\chi>>1$, KMC 
have shown that the growth times for these instabilities are respectively $\frac{t_{K H}}{t_{c c}}=\frac{v_{b} / v_{r e l}}{k a_{0}}$ and $\frac{t_{R T}}{t_{c c}}=\frac{1}{\left(k a_{0}\right)^{1 / 2}}$. These results indicate that the cloud will be substantially destroyed on timescales related to the cloud crushing time. As we shall see, this has important implications for laboratory laser experiments that will attempt to follow the shock-cloud evolution. A final timescale of importance to the shock-cloud interaction is the time for significant pressure variation behind the strong shock $t_{p}$. For a Sedov-Taylor blast this is

$t_{p}=\left|\frac{\partial \ln P}{\partial t}\right|^{-1} t \cong 0.2 t \cong \frac{0.1 R_{c}}{v_{b}}$

where $t$ is the age of the blast wave, $R_{c}$ is the distance of the cloud from the blast, and $v_{b}=\frac{d R_{b}}{d t}$ is the velocity of the blast wave. If the blast wave does not weaken significantly over the time for the cloud to be crushed and destroyed, $t_{c c}<t_{p}$. This constraint implies that the radius of the cloud $a_{0}$ satisfies $a_{0}<<0.1 R_{c} / \chi^{1 / 2}$. This is known as the small cloud limit. If on the hand, the blast wave does not change significantly as it sweeps over the cloud, but does evolve somewhat during the time it takes to crush the cloud than $t_{c c} \geq t_{p} \geq t_{i c}$ and this corresponds to cloud radii intermediate between $0.1 R_{c} / \chi^{1 / 2}$ and $0.05 R_{c}$. This is the intermediate cloud regime. As we shall see, our experiment is in this regime. In this case, the cloud will feel an impulsive force from the blast wave as the pressure decreases behind the blast wave shock.

\section{EXPERIMENTAL SETUP}

Strong shock waves produced in a radiation driven $\mathrm{CH}$ target by laser produced $\mathrm{x}$-rays were driven through a copper sphere. The motion and growth of the interaction were 
measured using shadowgrams produced by a large area x-ray backlighter produced by laser light. The sphere became hydrodynamically distorted after the passage of the shock, having a complicated down-stream structure. This was an instability-induced structure which was predicted by calculations. The experiment is a convenient laboratory analogue of the interactions occurring between supernova driven shocks and interstellar clouds. In addition, the experiment is important for providing validation data for three-dimensional codes.

A schematic description of the experiment is shown in Figure 2. The diagram is not to scale, and the area of the target package is greatly expanded. Eight Nova laser beams were focussed into a cylindrical gold hohlraum (Lindl 1995) producing a broad spectrum of x-rays which ablated the surface of the brominated plastic. The bromine dopant was included in the plastic to prevent preheat from high energy $\mathrm{x}$ rays produced in the hohlraum. The relatively planar shock wave produced by the ablation traveled down the cylinder, passing the copper sphere. Two additional laser beams impinged on a backlight target (titanium) and produced line radiation at about $4.5 \mathrm{keV}$, which passed through the cylinder perpendicular to its axis and were detected by the imaging camera. Differences in optical density to the backlight $\mathrm{x}$ rays formed a shadowgram which allowed positional information on the shock front and the sphere to be obtained.

Titanium was chosen as the backlight because its characteristic radiation at $4.5 \mathrm{keV}$ is completely stopped by even small amounts of copper, which allows accurate imaging of the breakup of the copper spheres. Also, there is a clear difference in absorption at this photon energy between the shocked and unshocked plastic. Eight of the ten Nova laser beams were used to heat the hohlraum and the two remaining beams were defocussed on the backlight target to produce a radiation source which was oval shaped with a diameter of about $800 \mu \mathrm{m}$. Since the objective was to measure the positions of both the shock front and the sphere as a function of time, the delays of the backlight beams were varied from 20 to $70 \mathrm{~ns}$ after the 
beginning of the laser pulse. The camera was opened $0.5 \mathrm{~ns}$ after the start of the backlight beams. The heating laser pulse width was $1.0 \mathrm{~ns}$ in length, and the backlight pulse widths were 3.0 ns. Using a standard Nova diagnostic, the flexible x-ray imager (Budil et al. 1996), four time snapshots could be taken during the backlighter pulse. It was necessary to make a correction for parallax to the positions measured on the film, as there were significantly varying angles between the axis of the camera and the line between the strips and the target. The data were extracted from the film after digitizing and analyzing the digitized data using a two-dimensional image analysis program (D. W. Phillion 1996, private communication).

\section{HYDRODYNAMIC SCALING}

In order that the laboratory experiment of the evolution of the interaction of a strong shock with a dense homogeneous copper ball be self-similar to the evolution of the interaction of an interstellar shock from a supernova blast wave with an interstellar cloud, several criteria must be satisfied. First, the laboratory and the astrophysical systems must both have a similar dimensionless shape as well as approximately equal values of a similarity parameter (Ryutov et al. 1999). In previous work (Klein at al. 1994) we have shown in detail that for strong shock-cloud interactions in the interstellar medium, the Euler equations of hydrodynamics exhibit Mach scaling. Thus if two systems are similar in all respects, but differ in the strength of the initial blast wave, the two systems will evolve identically when the initial shock is strong (of order Mach number = 10). In both the astrophysical environment as well as the laboratory experiment, we have assumed that the initial cloud is spherical and the shocks are strong, each having Mach numbers exceeding 10. The other dimensionless similarity variable shown to govern the evolution of the shocked cloud system is the density ratio of the cloud to the intercloud medium (Klein et al. 1994). In both the astrophysical interaction and in the laboratory experiment, the density contrast $\chi$ is taken to be 10. As Klein et al.(1994) have shown, these two dimensionless quantities allow the Euler equations of hydrodynamics 
to be scale invariant. These criteria are essentially the same as the criteria described by Ryutov et al. (1999) for strongly driven systems. We require that the initial density distributions are similar and also that the temporal dependence of the drives are similar for both the laboratory and the astrophysical systems. Since both systems have density contrast around 10 and the blast wave Mach numbers are similar $(M=10)$, these two criteria are met.

A key second requirement for hydrodynamic scaling from the astrophysical to the laboratory environment is that the Euler equations themselves must be a valid description of the hydrodynamic flow. This has been examined in detail by Klein et al. (1994) for the shock-cloud interaction in the ISM where the validity of the Euler equations was demonstrated. Here we examine this in the context of the lab experiment. As pointed out in Ryutov et al. (1999), four criteria must be established for the ideal Euler equations to be a valid description. These criteria are: (1) the system needs to be collisional; (2) the effects of thermal conduction should be negligible; (3) the effects of radiation should be negligible; and (4) viscous dissipation needs to be insignificant. Here we examined these constraints for both the $\mathrm{Cu}$ ball and the $\mathrm{CH}$ background for physical conditions prevalent in the laboratory experiment. We use the analysis of Ryutov (1999) for examination of the criteria. A key criterion for the validity of the Euler equations for our experiment is that the plasma be in the collisional regime. Thus the collisional mean free path of the iions $l_{c}$ should be small compared to the characteristic scale length of the problem $h$ which we take to be 100 microns, approximately the initial radius of the $\mathrm{Cu}$ ball. For conditions that are characteristic of the experiment we have $\mathrm{T}=5 \mathrm{eV}$ and density $8.9 \mathrm{~g} / \mathrm{cm}^{3}$ for $\mathrm{Cu}$ and $\mathrm{T}=10 \mathrm{eV}$ and density $1.06 \mathrm{~g} / \mathrm{cm}^{3}$ for $\mathrm{CH}$. We estimate the ratio of the collision mean free path $l_{c}$ to the characteristic spatial scale $h$ to be

$$
\frac{l_{c}}{h} \cong \frac{3 \times 10^{13}[T(e v)]^{2}}{\Lambda h n_{i}}
$$


where $\Lambda$ is the Coulomb logarithm and taken to be $\sim 6$ and $n_{i}$ is the ion density. We find that $l_{c} / h=1.5 \times 10^{-7}$ for $\mathrm{Cu}$ and $1.0 \times 10^{-6}$ for $\mathrm{CH}$. Thus ions are well localized and the plasma is collisionally dominant. We next examine the efficiency of transport of energy by thermal conduction by evaluating the Peclet number Pe. For thermal energy transport conduction to be negligible, we require that $\mathrm{Pe}=h v / \eta \gg>1$ where $v$ is a typical flow velocity and $\eta$ is the thermal diffusivity for electrons. We estimate the thermal diffusivity as in Ryutov et al (1999 where

$$
\eta=\frac{3.3 \times 10^{-3} A[T(e v)]^{5 / 2}}{\Lambda Z(Z+1) \rho\left(g / \mathrm{cm}^{-3}\right)}
$$

and $\mathrm{A}$ and $\mathrm{Z}$ are the atomic weight and charge of the ions.

We find that $\mathrm{Pe}=8.0 \times 10^{7}$ and $4.0 \times 10^{5}$ for $\mathrm{C}$ and $\mathrm{CH}$ respectively. Thus convective transport greatly exceeds thermal conduction and we can neglect thermal conduction. We next examine the radiation flux. For scaling to be valid we must show that the energy flux carried by radiation must be small compared to the hydrodynmic fluxes, thus no additional scales enter the problem. We estimate the mean free path of the photons to be the minimum of the mean free path due to inverse bremsstrahlung and Compton scattering (Ryutov et al. 1999). For typical densities and temperatures in the $\mathrm{Cu}$ and $\mathrm{CH}$ we easily find that the photon mean free path in both fluids is far less than the characteristic spatial scale of the system $h$, thus the system is relatively optically thick. In this limit, a sufficient condition that the radiative losses are negligible is that the radiative cooling time $\tau_{c}$ greatly exceed the characteristic hydrodynamic time. A characteristic hydrodynamic timescale is the cloud crushing time $t_{c c}$ which for our experiment is $\sim 8 \times 10^{-9} \mathrm{sec}$. It can be shown (Ryutov et al. 1999) that the cooling time is

$$
\tau_{c}=\frac{0.7(Z+1) \rho_{i} h}{A[T(e v)]^{3}}
$$


We find that in $\mathrm{Cu} \tau_{\mathrm{c}}=2.37 \times 10^{-4} \mathrm{sec}$ and in $\mathrm{CH} \tau_{\mathrm{c}}=4.08 \times 10^{-6} \mathrm{sec}$. Thus the cooling time greatly exceeds the characteristic hydrodynamical time and the energy fluxes carried by radiation are indeed negligible. Finally, for the Euler equations to apply we require that viscous effects be negligible. Taking into account all sources of the kinematic viscosity including both the photon viscosity and the particle viscosity in the collisional limit we find that the Reynolds number of the flow is a least $10^{6}$, thus the viscous forces are small compared to the inertial forces and can be neglected. Having established the four criteria stated above, we are well within the regime where the Euler equations of gas dynamics apply to our system and our scaling arguments are satisfied.

\section{RESULTS OF HYDRODYNAMIC SIMULATIONS}

In order to simulate the evolution of the interaction and compare with the results to the NOVA experiments, we performed a series of $2 \mathrm{D}$ radiation-hydrodynamic calculations using an arbitrary Lagrangian-Eulerian multi-fluid radiation-hydrodynamics code (CALE) that allows realistic equation of state data (cf. Kane et al. 1997). CALE uses an operator split methodology that employs second order accurate monotone advection with interface reconstruction to track material interfaces followed by a Lagrangian re-map step using predictor-corrector time stepping with monotonic artificial viscosities. An advantage of this approach is to allow adaptivity to occur when needed through a Lagrangian grid, and fixed Eulerian grids to occur when grid cells are distorted to an extent that compromises accuracy. Most of the calculations we describe in this paper have been made with fixed Eulerian grids with the Lagrangian option turned off to compare with previous high resolution fixed grid Eulerian calculations we performed with adaptive mesh refinement (Klein et al. 1994). 
The calculations were set up initially so that the copper sphere remained in pressure equilibrium with the surrounding $\mathrm{CH}$. A series of one dimensional radiation-hydrodynamic calculations using laser deposition was first performed to model the strong shocks that are produced by the X-ray irradiated $\mathrm{CH}$ target (cf. figure 2.) From these 1-D calculations, we developed a time dependent temperature profile that when applied as a boundary condition on the boundary of the $\mathrm{CH}$ ablator, drove a shock wave of the correct shock speed down the tube. Calculations were initially set up with a fixed Eulerian grid such that the grid resolution was 50 cells per initial sphere radius $\left(R_{50}\right)$ in a region surrounding the sphere that was taken to be 2.5 sphere radii in the radial direction and 6 sphere radii in the axial direction. This region, which was 125 cells by 300 cells was large enough to capture the full motion of the sphere during its evolution for all times of interest in the experiment and allowed the sphere to be captured with $R_{50}$ resolution throughout its trajectory in the background fluid. This resolution $\left(R_{50}\right)$ was determined to represent a reasonably converged simulation as demonstrated by a detailed convergence study of several global quantities, e.g. mean density, rms velocity, radial and axial distortion (Klein et al.1994). Using the convergence scaling established in Klein et al. (1994) we determined that we are within $10 \%$ converged on many of these quantities at the present resolution of $R_{50}$. The computational domain of the 2-D calculations included the full 700 micron by 2000 micron domain encompassed by the actual experiment. Although the region well outside the sphere was taken to have only $R_{25}$ resolution, and this resolution is clearly degraded from the region surrounding the sphere during its evolution, it was determined that it is only crucial to have reasonably high resolution in the region surrounding the sphere at all times to adequately represent the hydrodynamic instabilities that are significant in the destruction of the sphere.

In Figures 3 through 9, we present 2-D (axisymmetric) images of the evolution of the density field of the shocked copper sphere as it moves in the background $\mathrm{CH}$. For our 
purposes, the sphere is representative of an interstellar cloud that has been impacted by a supernova blast wave and is moving through the inter-cloud medium (ICM). In our NOVA experiment, the initial density contrast between the sphere and the background was about 8 , which is comparable to the density contrast one can expect for a variety of interstellar clouds and the ICM. The shock in the experiment was strong with a Mach strength of about 10, comparable to the strength of a supernova shockwave. The key four stages that have been identified in the shock-cloud interaction (Nittman.et al. 1982, Klein et al. 1994) can all be identified in the shock sphere interaction NOVA laser experiment. They are (i) the initial development of reflected, transmitted and diffracted shocks, (ii) shock compression, convergence and multiple shock interaction resulting in vorticity production and appearance of triple points in the shocked flow, (iii) re-expansion of the cloud into the ICM and subsequent shear flow instabilities and (iv) eventual destruction by resultant hydrodynamic instabilities and differential forces. As we have shown in section 4, the shock-cloud interaction is expected to self-similarly scale from the characteristic parsec scales of the interstellar clouds down to the micron scales of the NOVA experiment.

In Figure 3 we show a color image of the density at a time 22 nanoseconds ( $n s$ ) after the laser has first turned on. The abscissa is the symmetry axis and we only compute the upper half of the symmetry plane in this $\mathrm{r}-\mathrm{z}$ axisymmetric calculation. Using equation 2.4 we find that the crushing time $\left(t_{c c}\right)$ is about $8.3 \mathrm{~ns}$ for this experiment. Several features of the shockcloud interaction are evident at this time. Since the initial blast wave first interacts with the sphere at about $18 \mathrm{~ns}$, the sphere has evolved for $0.48 t_{c c}$ since the initial shock interaction. The initial ablation driven shock is seen to have moved about $60 \%$ into the copper sphere shocking the initial copper up by more than a factor of 2 . A transmitted shock is driven into the sphere at a velocity that is approximately a factor $3 / 4$ smaller than the initial blast wave velocity of the shock. A weak reflected bow shock is seen to move away from the surface of 
the shocked sphere. We also note a further shock in the Be tamper that lags the initial ablation shock. At 24 ns (Fig. 4) the initial ablation shock has diffracted around $90 \%$ of the sphere's surface. This sweeping of the shock around the surface of the sphere has resulted in the a shear flow between the surrounding $\mathrm{CH}$ material and the copper sphere material. This results in the start of a Kelvin-Helmholtz $(\mathrm{K}-\mathrm{H})$ instability at the side of the sphere as we note the formation of a small arm on the side of the cloud. By $26 \mathrm{~ns}\left(0.96 t_{c c}\right)$ the initial ablation shock has traversed the surface of the sphere. The sphere is undergoing considerable axial crushing at this time with clear evidence of the $\mathrm{K}-\mathrm{H}$ instability present at the side of the sphere (Fig. 5). At $30 \mathrm{~ns}\left(t_{c c}=1.44\right)$ the ablation shock has moved well ahead of the $\mathrm{Cu}$ sphere (Fig. 6). This shock has undergone a complex double Mach reflection resulting from a shock-shock interaction on the symmetry axis. We note the appearance of a Mach shock disk, and a Mach reflected shock connected by a triple point in the flow. The Mach reflected shocked interacts with the transmitted shock in the sphere re-shocking the $\mathrm{Cu}$ sphere and further compressing it. The K-H instability has resulted in a side arm of the sphere stretching out in the direction of the initially propagating ablation shock. The initial highly compressed sphere undergoes some de-compression as the sphere begins to expand radially. At $43 \mathrm{~ns}\left(t_{c c}=3.0\right)$ we see the continuing stretching out of the sphere and the wind up of the stretched arms due to $\mathrm{K}-\mathrm{H}$ instabilities (Fig. 7). It is important to note that at this time the sphere is still relatively intact and although highly deformed from its initial spherical configuration, it is not broken up. Much later in the evolution at $50 \mathrm{~ns}\left(t_{c c}=3.85\right)$, the sphere has undergone considerable radial expansion and axial stretching (Fig. 8). Strong K-H instabilities have begun to shred the cloud along its sides. The sphere's arms have begun to wind up on the back side and this has resulted in a large increase in baroclinic vorticity and the development of a vortex ring in 3D (cf. Klein et al. 1994). The development of a vortex ring and its associated non-axisymmetric instabilities in $3 \mathrm{D}$ will have important 
consequences for the late time evolution of the sphere and the related evolution of shocked interstellar clouds. The radiographic imaging of this vortex ring requires face-on radiography of the evolving cloud (sphere). Simultaneous face-on and side on radiography is not possible on the indirect drive NOVA laser. This has been achieved for the first time in a set of experiments conducted recently on the OMEGA laser with indirect drive (Klein et al. 2002, Robey et al. 2002). These experiments have uncovered the modal structure of the vortex ring and have shown that the breakup of the ring can be explained with detailed 3-D numerical simulations. The modal structure of the ring was shown (Klein et al. 2002, Robey et al. 2002) to be in excellent agreement with that predicted by application of incompressible theory for non-axisymmetric instabilities in a vortex ring (Widnall et al. 1974). Finally at 54 $\mathrm{ns}\left(t_{c c}=4.3\right)$ the sphere is undergoing severe $\mathrm{K}-\mathrm{H}$ instabilities as small pieces of the cloud separate from the main body in the stretched out arms (Fig. 9). Although pieces of the cloud have begun to separate from the main body due to $\mathrm{K}-\mathrm{H}$, the $2 \mathrm{D}$ axisymmetric calculations indicate that the cloud as a whole remains intact and that its geometry is like a thinly drawn out shell.

In order to directly compare the results of the detailed $2 \mathrm{D}$ calculations with the experimental images, it is important to generate simulated absorption radiographs of the $2 \mathrm{D}$ density field generated by our simulations. This is done by performing a radiative transfer ray trace calculation through the rotated, axially symmetric calculated density field using the opacity of the copper and the surrounding $\mathrm{CH}$ to $\mathrm{x}$-ray line radiation at $4.5 \mathrm{keV}$. The simulated radiographs are an excellent approximation of the $\mathrm{x}$-ray images that are generated by the laser experiment as two of the laser beams are used to backlight a Titanium target. They produce line radiation at about $4.5 \mathrm{keV}$ that subsequently passes through the cylindrical target chamber perpendicular to its axis and forms an X-ray shadowgram after impinging on the copper sphere and through the surrounding $\mathrm{CH}$ material. In the next figures at $43 \mathrm{~ns}$ and 
$54 \mathrm{~ns}$ corresponding to $3 t_{c c}$ and $4.3 t_{c c}$ respectively, we show the simulated radiographs from our $2 \mathrm{D}$ calculations at times when the copper sphere has undergone considerable crushing corresponding to late times in the evolution of a shocked cloud. In Figure 10 we show a simulated absorption image of the sphere at $43 \mathrm{~ns}\left(\mathrm{t}=3 t_{c c}\right)$. The simulated $\mathrm{x}$-ray radiograph indicates that the sphere is essentially intact and shows no signs of fragmenting. The upper half plane computed radiograph has been symmetrically imaged to the lower halfplane for comparison with experimental data. At $54 \mathrm{~ns}\left(\mathrm{t}=4.3 t_{c c}\right)$ the simulated radiograph again appears to be mostly opaque to the absorbing radiation of the x-ray backlighter (Fig. 11). In Figure 12 we display a one-dimensional cut through the central plane of the simulated X-ray absorption radiograph at $43 \mathrm{~ns}$ revealing the X-ray absorption profile of copper sphere and the surrounding lower density $\mathrm{CH}$. Here we see that the sphere, located between 965 microns and 1075 microns, is almost $100 \%$ absorbing with a small amount of transmission at the $2-3 \%$ level. The profile of absorption for the simulation is in stark contrast with a one-dimensional cut through the experimentally measured radiograph shown on the same plot. The experimental measurement indicates that over $50 \%$ of the sphere has a significant reduction in absorption which as much as $30 \%$ transparency to the $4.4 \mathrm{keV} \mathrm{x}$-rays. At $54 \mathrm{~ns}$ we show similar one-dimensional cuts of the absorption through the simulated and experimentally determined radiographs (figure 13). The amount of this transparency is clearly displayed in a one-dimensional cut (Fig. 13). The central region of the sphere has a transmission of about $5 \%$, but the bulk of the sphere remains intact and is highly absorbing to the incident radiation. These radiographic images and the associated density fields indicate that even at late times in the evolution of the shocked sphere, the developing instabilities are not severe enough to destroy the sphere. Extending this scenario to the case of the shocked interstellar cloud, one might conclude that clouds may undergo significant distortion due to strong blast waves, but they would inevitably stay intact. The caveat is of course that these 
calculations have assumed that the shocked cloud is axisymmetric and can be reasonably modeled by $2 \mathrm{D}$ calculations. How reasonable an approximation this is will depend on what types of instabilities may be present in $3 \mathrm{D}$ flow that has no counterpart in $2 \mathrm{D}$, and furthermore, how important such instabilities might be in dominating the evolution of the cloud. The test of how well 2D axisymmetry does in representing this interaction will be a direct comparison against experimental results.

\section{EXPERIMENTAL RADIOGRAPHS}

We have imaged the evolution of the shocked sphere through a series of time snapshots by the methods described in section 2. Using scaling arguments described in section 4. and referring to our evolution terms of crushing times, $t_{c c}$, we can use these experimental radiographs to explore the actual evolution that a shocked interstellar cloud would undergo at similar elapsed times.

Figure 14 shows a series of shock-sphere shadowgrams obtained from times from $20 \mathrm{~ns}$ to $26.5 \mathrm{~ns}$ after the laser pulse and show the early time crushing of the sphere. Taking into account that the time the shock first reaches the sphere, these images show the initial crushing of the cloud from $0.24 t_{c c}$ to $1.02 t_{c c}$. In this series, we clearly note the propagation of the Mach 10 shock through the sphere. The arrows show the shock positions. The sphere undergoes significant compression and shows the initial behavior of KelvinHelmholtz instabilities around the sides of the sphere at about 26 ns due to shear flow between the sphere and the surrounding plastic, induced by shock diffraction around the sides. The evolution represents the initial development of reflected, transmitted and diffracted shocks in the first stage of the shock-cloud interaction. 
In figure 15 we show a full panel of side-on x-ray radiographs tracing the evolution of the shocked cloud after the passage of the initial blast wave that moves down through the cloud from the upper vertical position in each frame of the panel. The panel extends from $30.2 \mathrm{~ns}$ on the upper left out to $61.2 \mathrm{~ns}$ on the lower right. It should be noted that in order to get such a large dynamic range in time snapshots in the laser experiment, we must perform several laser shots, thus this evolution represents a set of consecutive laser experiments spanning the full range in time. A consequence of this procedure is that it is not possible to get uniform conditions of the quality of data throughout all the laser shots in the series.

This can also be seen in Figure 16 where we plot the positions of the shock and ball as a function of time. If conditions were exactly uniform on every shot, shock positions and sphere positions would lie on nearly straight lines. Small differences in laser drive energy and manufacturing tolerances give rise to the spread in the data. The shock positions were measured on a vertical line through the center of the sphere. This was done because there was some variation in the shock position in the lateral direction. The sphere position was that measured for the center of the spheres, even though in many cases later in time, the sphere was definitely not spherical. The velocity of the sphere in the background foam is in reasonable agreement with the simple drag theory developed by Klein et al. (1994).

Returning to the discussion of Figure 15 we note that at At 30.2 ns, the initial spherical cloud has undergone substantial compression in the axial direction and has started to expand radially. By $36.7 \mathrm{~ns}$ this is clearly noticeable as the cloud had expanded greater than a factor of 2 in the radial direction and the development of extended arms at the sides of the cloud are beginning to develop due to roll up of the side of the cloud by differential shear flow with the inter-cloud medium, in this case the $\mathrm{CH}$ foam. The structure has taken on an "umbrella" shape. This shape occurs during the compression phase. As shown by Klein et al. 1994, the flow around the cloud converges on axis behind the cloud, producing a high-pressure 
reflected shock in the inter-cloud medium and driving a shock into the rear of the cloud. The shocks compressing the cloud from the sides are weaker than those at the front and back because the pressure is a minimum at the sides. Hence during this phase the sphere is compressed as a pancake. At this time the cloud has entered the second stage of the shockcloud interaction with shock compression, convergence and multiple shock interaction resulting in vorticity production and formation of triple points in the shocked flow. At the present time we have not be able to image the presence of triple points in the flow as this will require doping the initial copper sphere with another material. The production of a large vortex ring at the back part of the cloud results in the initial winding up of the side arms of the cloud.

The third phase of the shock-cloud evolution, re-expansion of the cloud into the ICM and subsequent shear flow instabilities is well underway by $43 \mathrm{~ns}$. At this time and more clearly at $49 \mathrm{~ns}$ corresponding to $3.7 t_{c c}$ the cloud has apparently undergone a severe hydrodynamic instability and shows a significant separation between the front (top) and the back (bottom). This separation results in a voiding in the sphere. The $2 \mathrm{D}$ calculation does a good job in getting the overall morphology of the sphere correct at this time, but indicates no such separation of the front and back of the sphere in the simulated x-ray radiograph.

The $2 \mathrm{~d}$ calculation also does a good job of calculating the outer envelope of the hydrodynamic evolution of the sphere. In Figure 17 we show a comparison between calculation and experiment for the sphere height. By height we mean the dimension of the sphere parallel to the direction of the shock. As the shock passes the sphere, the sphere is first compressed and then later expands. The degree of compression and expansion is in reasonable agreement with experiment. In Figure 18 we plot the width of the sphere as it expands after the passage of the shock. Again there is reasonable agreement between calculation and experiment. 
Properly modeling the detailed break-up of the sphere requires 3D calculations. Preliminary 3D AMR simulations (Klein and McKee 1994) of the shock-cloud interaction for a Mach 10 shock interacting with an interstellar cloud that has a density ratio of 10 with the surrounding ICM first showed that the shocked cloud undergoes severe non-axisymmetric instabilities not existent in 2D. This results in a fluted structure in the lower half of the cloud (Figure 19). The 3D AMR simulations had a resolution of $R_{90}$ (90 cells per initial sphere radius). The lower part of the sphere has a diameter of 5 initial sphere radii, thus the fluted structure was about 450 cells across. The structure is more highly fragmented and considerably less coherent than in $2 \mathrm{D}$ at a comparable time. As we pointed out, the $2 \mathrm{D}$ calculation has a resolution that is reasonably well converged, thus the significant differences in the 2D and 3D calculations appear to be due to real physical effects, not due to lack of resolution. In 3D the cloud undergoes azimuthal bending mode instabilities in a powerful vortex ring that forms in the back part (bottom) of the shocked sphere (Klein et al. 2000; Marinak, Klein and Perry 2001). This is analogous to the Widnall instability in incompressible flow. This instability results in the breaking up of the strong vortex ring associated in the intercloud medium surrounding the sphere, into smaller less coherent vortex structures. The 3D results indicate that this breaking up of the vortex ring by this bending mode instability results in the voiding in the sphere in good agreement with the experiment (Marinak, Klein and Perry 2001). Coherent stable vortex rings found in 2D simulations become unstable in 3D and would result in more effective mixing and turbulence in 3D. The transition to turbulence in the evolution of the sphere can be seen at $55 \mathrm{~ns}$. By 61 ns the sphere has become fully turbulent leading to its full destruction. An elucidation of the dynamical properties of the vortex ring and the role of non-axisymmetric instabilities in vortex rings causing the bifurcation of the sphere requires careful 3D calculations with realistic equations of state. These calculations will be discussed in a separate paper. 


\section{COMPARISON WITH OBSERVATIONS}

Our experiment is showing the first evidence of 3D bending mode instabilities in shocksphere interactions at high Mach number. The crucial aspects of this instability can be understood by high-resolution 3D hydrodynamic calculations, but are not well represented by 2D calculations. Later time evolution of the shock-sphere interaction experiment shows the transition from the breakup of the sphere due to bending mode and Kelvin Helmholtz instabilities to the turbulent regime with structure present on a wide range of scales. These experiments are potentially promising in understanding the interaction of supernova blast waves with interstellar clouds. KSM demonstrated that the physics of this interaction can be well understood by consideration of the density ratio of the cloud to the inter-cloud medium and the Mach number of the shock. They show that by consideration of these two parameters, the evolution of a sphere after interaction with a strong shock is scale invariant for the case of non-radiative shocks. They apply their calculations for a Mach 10 shock to the interaction of the supernova remnant in the Cygnus Loop with the Southwestern Cloud. By conducting a series of shock sphere laser experiments examining the sensitivity of the interaction to the variation of these parameters, we hope to be able to scale the experiments to actual structures in the Cygnus Loop. The greatly improved sensitivity and spatial resolution of the ROSAT-HRI $x$-ray observations and those of Chandra make it ideal for exploring blast wave-cloud interactions. The combination of these high-resolution observations with our Nova laser experiments and high-resolution 3D AMR calculations gives us a unique approach to studying this fundamental interaction in the ISM.

This work was performed under the auspices of the U.S. Department of Energy by the Lawrence Livermore National Laboratory under Contract No. W-7405-ENG-48. 


\section{REFERENCES}

Budil, K. S., Perry, T. S., Bell, P. M., Hares, J. D., Miller, P. L., Peyser, T. A.,Wallace,R.,

Louis, H., \& Smith, D. E. 1996, Rev. Sci. Instrum., 67, 485

Kane, J. et al. 1997, ApJ. 478, L75

Klein, R. I., Robey, H. F., Perry T. S., Kane, J. D., Greenough, J. A.,\& Marinak, M. 2001

IFSA, eds. K. A. Tanaka, J. Meyer-ter-Vehn, and D. Meyerhofer, in press

Klein, R. I., Budil, K. S., Perry, T. S., \& Bach, D. R. 2000, ApJS, 127, 379

Klein, R. I., Mckee, C. F., \& Colella, P. 1994, ApJ, 420, 213

Levenson, N. A. et al. 1997, ApJ, 484, 304

Lindl, J. 1995, Phys. Plasmas, 2 (11), 3933

McKee, C. F., Cowie, L. L., \& Ostriker, J. P. 1978 ApJ, 219, L23

Nittman, J., Falle, S., \&. Gaskell, P. H. 1982, MNRAS, 201, 833

Robey, H. F. et al. 2002, Phys. Rev. Lett., in press

Ryutov, D., Drake, R. P., Kane, J., Liang,E., Remington, B. A., \& Wood-Vasey, W. M. 1999, ApJ 518, 821

Widnall, S., Bliss, D. B., \& Tsai, C. Y. 1974, J. Fluid. Mech., 66, 35

Woodward, P. R., 1976, ApJ, 207, 484

Zabusky, N. M., Zeng, S. M., Ray, J. \&. Samtaney, R. 1997, Proc. of the

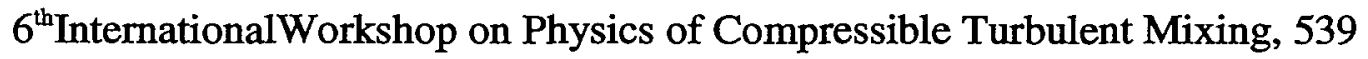

FIGURE CAPTIONS 
Figure 1: A schematic diagram showing the basic parameters used for defining the cloud crushing time.. At $t=0$ a shock approaches a spherical cloud of constant density $\rho_{i 0}$ whose initial radius is $a_{0}$ with a velocity $v_{b}$. The cloud is assumed to be in pressure equilibrium with the surrounding ambient medium. The interaction results in a reflected shock and transmitted shocks within the cloud. The initial shock eventually interacts with itself around the backside of the cloud resulting in a Mach reflected shock back into the cloud.

Figure 2: A schematic diagram of the initial set up of the experiment showing a cross section through a cylindrical target used for the experiment. The dimensions given on the package are nominal and were varied from experiment to experiment. The axis of the cylinder is vertical.

Figure 3: Color density image of the $2 \mathrm{D}$ axisymmetric calculation at $\mathrm{t}=22 \mathrm{~ns}$. The initial shock is still within the copper ball. Shock has come from the left side of the domain. The sphere was placed on the symmetry plane.

Figure 4: Color density image of the simulation at $t=24$ ns. The initial shock has diffracted around $90 \%$ of the sphere.

Figure 5: Color density image of the simulation at $t=26 \mathrm{~ns}$. The shock has passed over the sphere and the start of a shear flow instability around the sides of the sphere is seen. 
Figure 6: Color density image of the simulation at $t=30$ ns. The sphere has undergone substantial distortion. Mach reflected shocks are in evidence.

Figure 7: Color density of the simulation at $t=43 n s$.

Figure 8: Color density image of the simulation at $t=50 \mathrm{~ns}$. The sides of the sphere are Kelvin Helmholtz unstable. Region of a strong vortex region is noted.

Figure 9: Color density image of the simulation at $t=54$ ns. The sphere has undergone severe axial and radial distortion and has become a thin shell.

Figure 10: Simulated radiograph of the sphere at 43 ns. The radiograph has been rotated about the symmetry plane. The radiograph was computed with a ray trace radiative transfer calculation using the material opacities.

Figure 11: Simulated radiograph at 54 ns.

Figure 12: Comparison of the calculated $\mathrm{x}$-ray absorption profile from the simulation and the measured absorption from the experiment at $43 \mathrm{~ns}$.

Figure 13: Comparison of the calculated x-ray absorption profile from the simulation and the measured absorption from the experiment at $54 \mathrm{~ns}$. 
Figure 14: Shock-sphere shadowgrams. These were obtained at times from 20 to $26 \mathrm{~ns}$ after the laser pulse and show the early time crushing of the sphere.

Figure 15: Shock-sphere shadowgrams. These were obtained from $30.2 \mathrm{~ns}$ to $61.2 \mathrm{~ns}$. They illustrate the evolution of the sphere from its early crushing phase through the phase undergoing severe vortex ring instabilities to its final turbulent destruction at late time.

Figure 16: Results of the shock sphere experiment showing the position of the shock front and the position of the center of the sphere as a function of time after the beginning of the laser pulse. The zero of the ordinate is the position of the sphere before the beginning of the drive. The shock front was measured in a vertical line passing though the center of the sphere.

Figure 17: Comparison of the results of the shock sphere experiment and the 2D simulations showing the time evolution of the axial height of the sphere.

Figure 18: Comparison of the results of the shock sphere experiment and the 2D simulations showing the time evolution of the radial width of the sphere.

Figure 19: Volume rendered image of 3D AMR simulation of the sphere at late time. This calculation used an ideal EOS and is scale free. It shows the breakup of the sphere due to 
vortex ring instabilities. Note the bifurcation of the sphere and the multimode fluted structure 
$t_{0}=0$

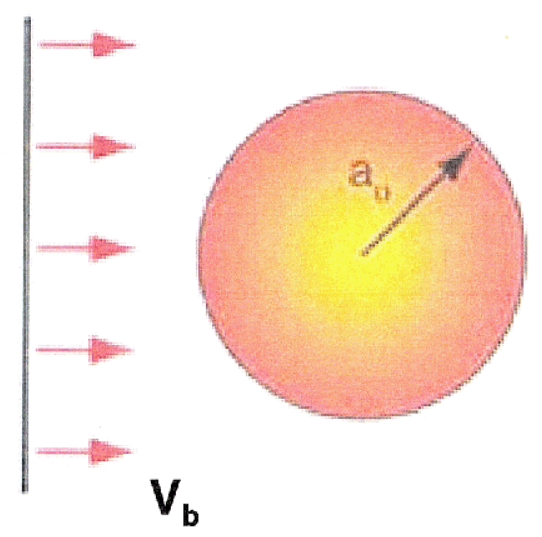

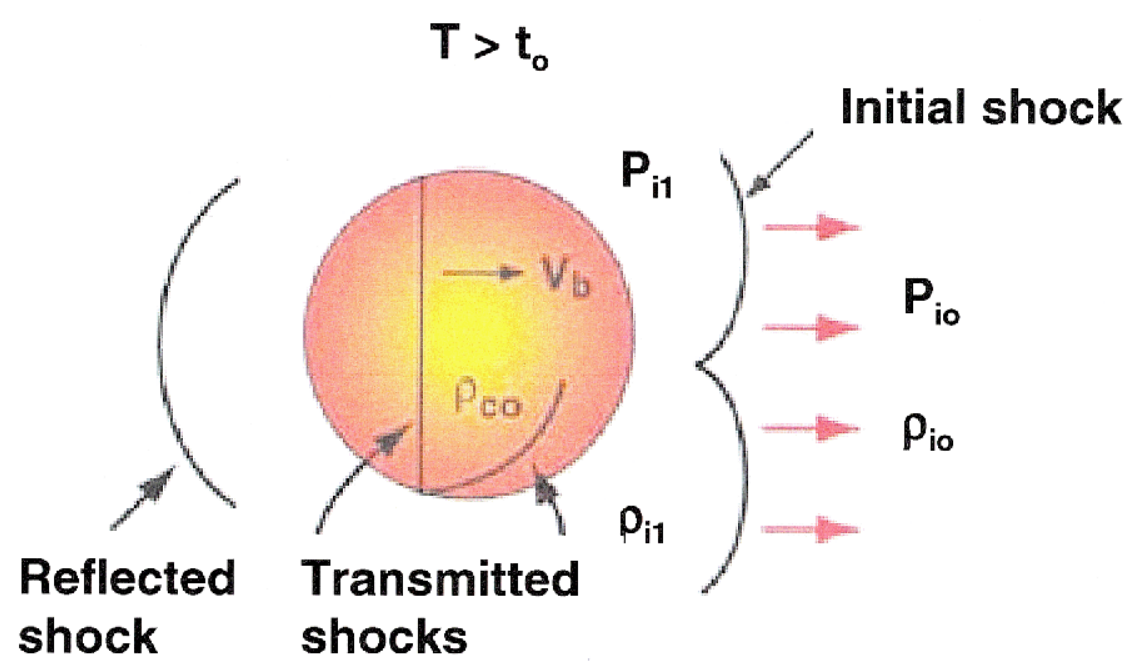

Figure 1 


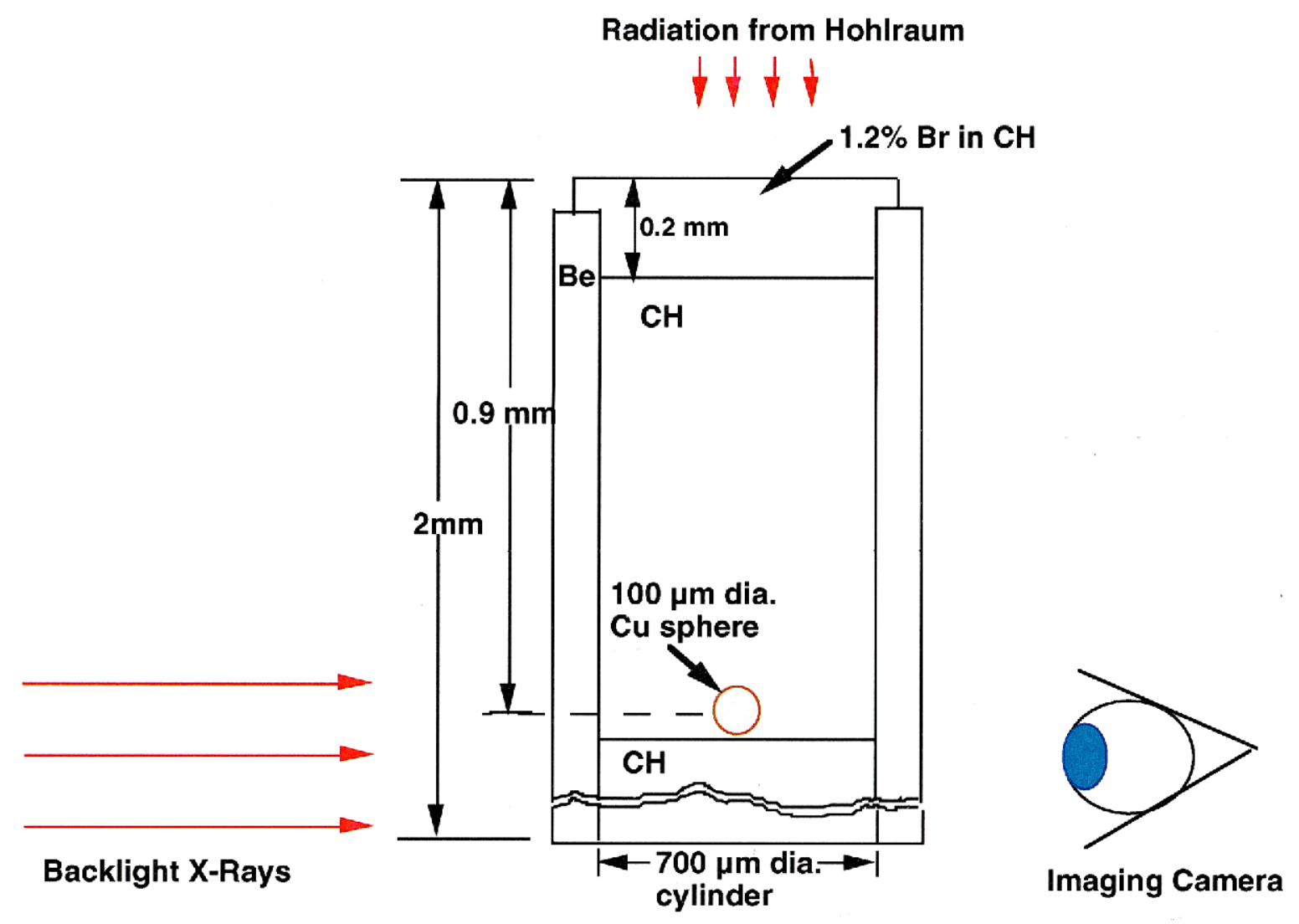

Figure 2 


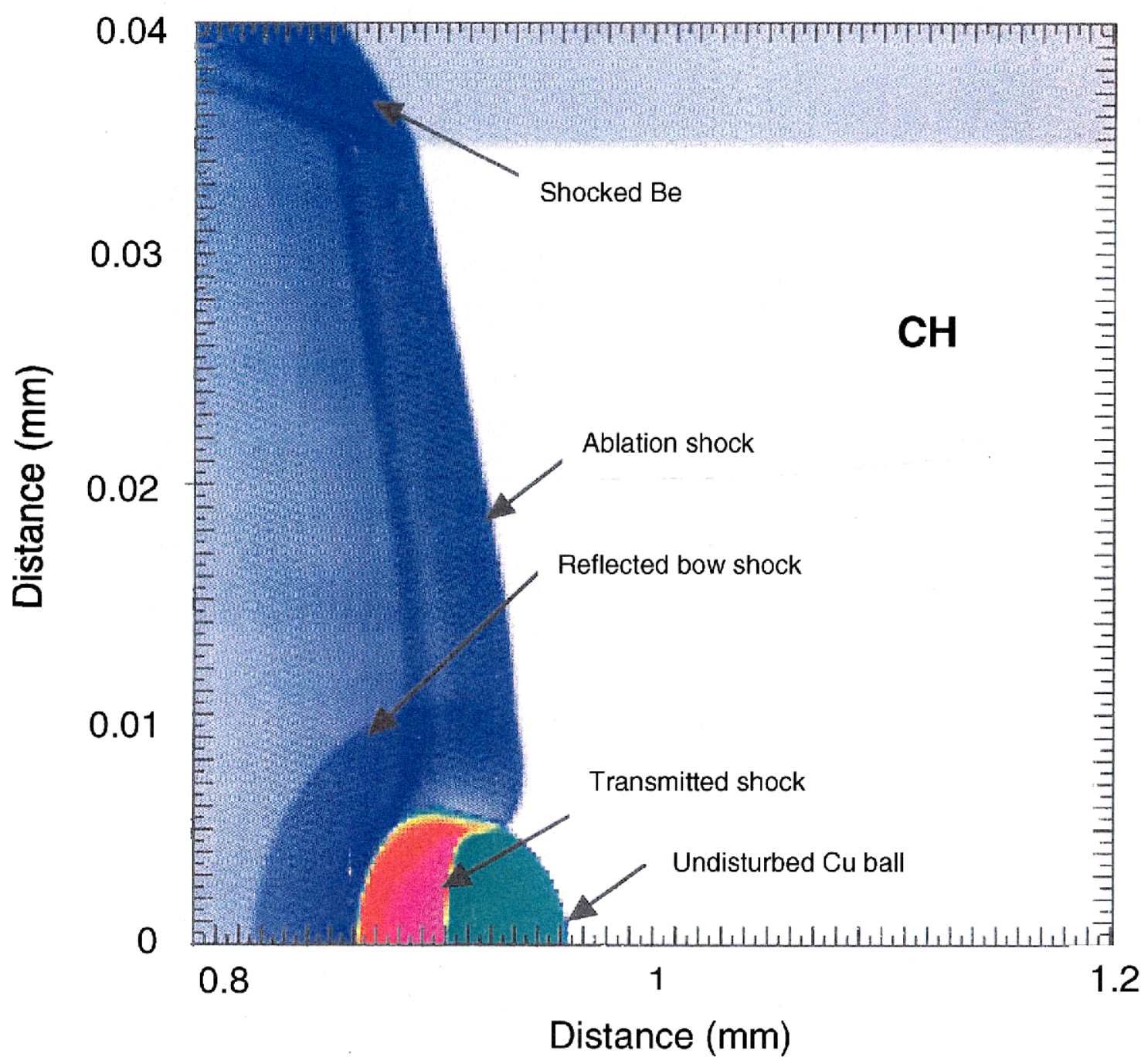

Figure 3 


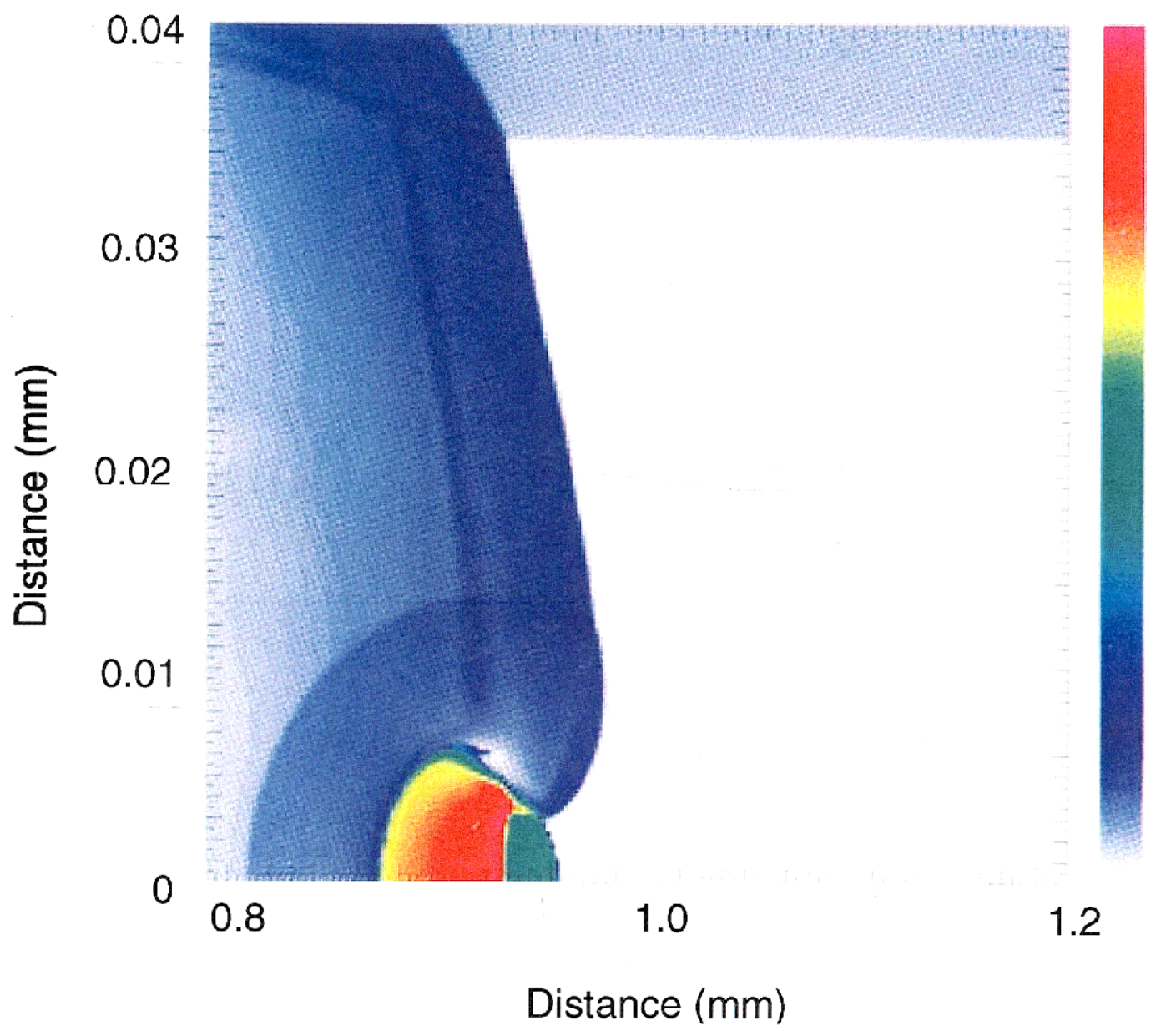

Figure 4 


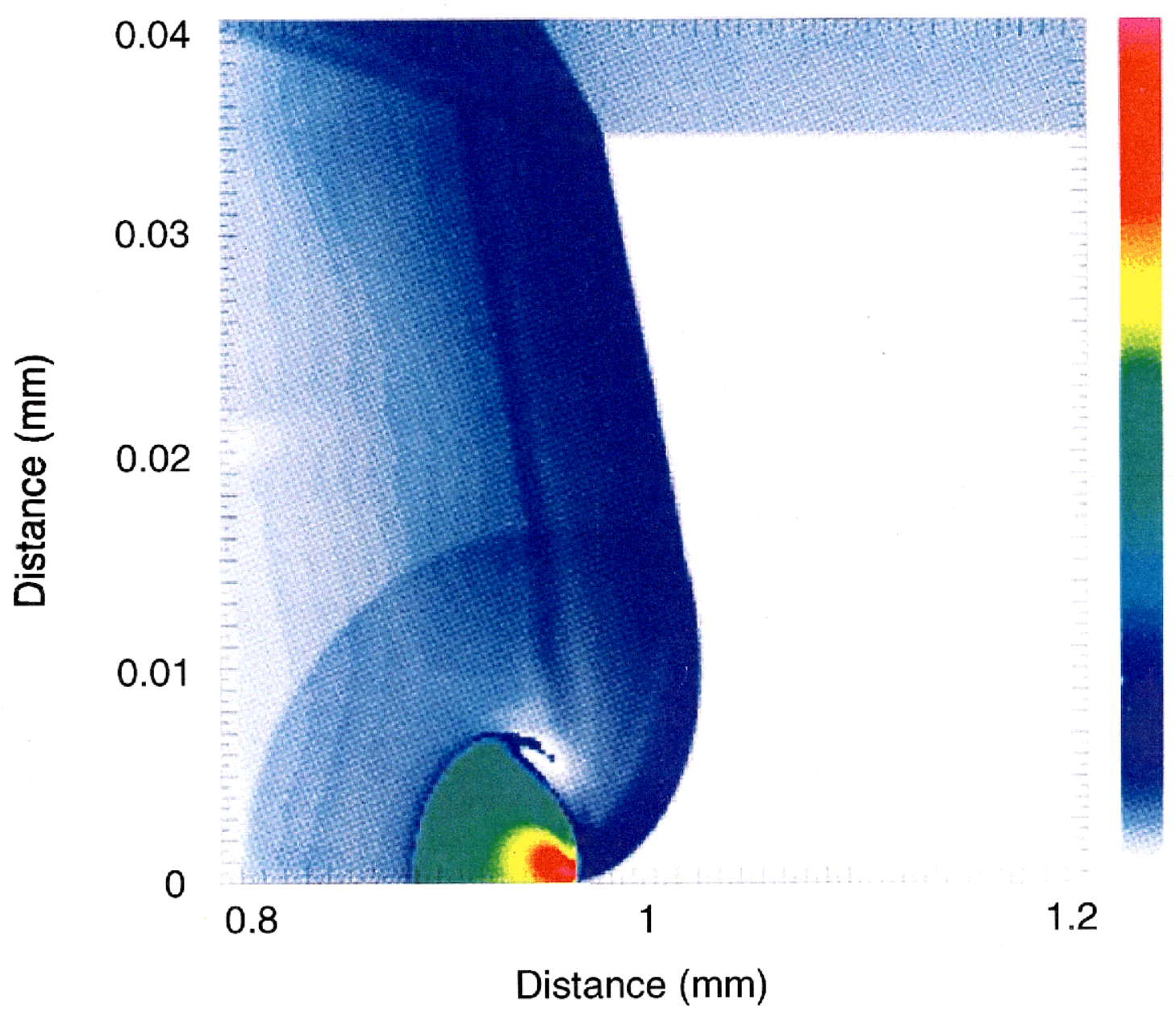

Figure 5 


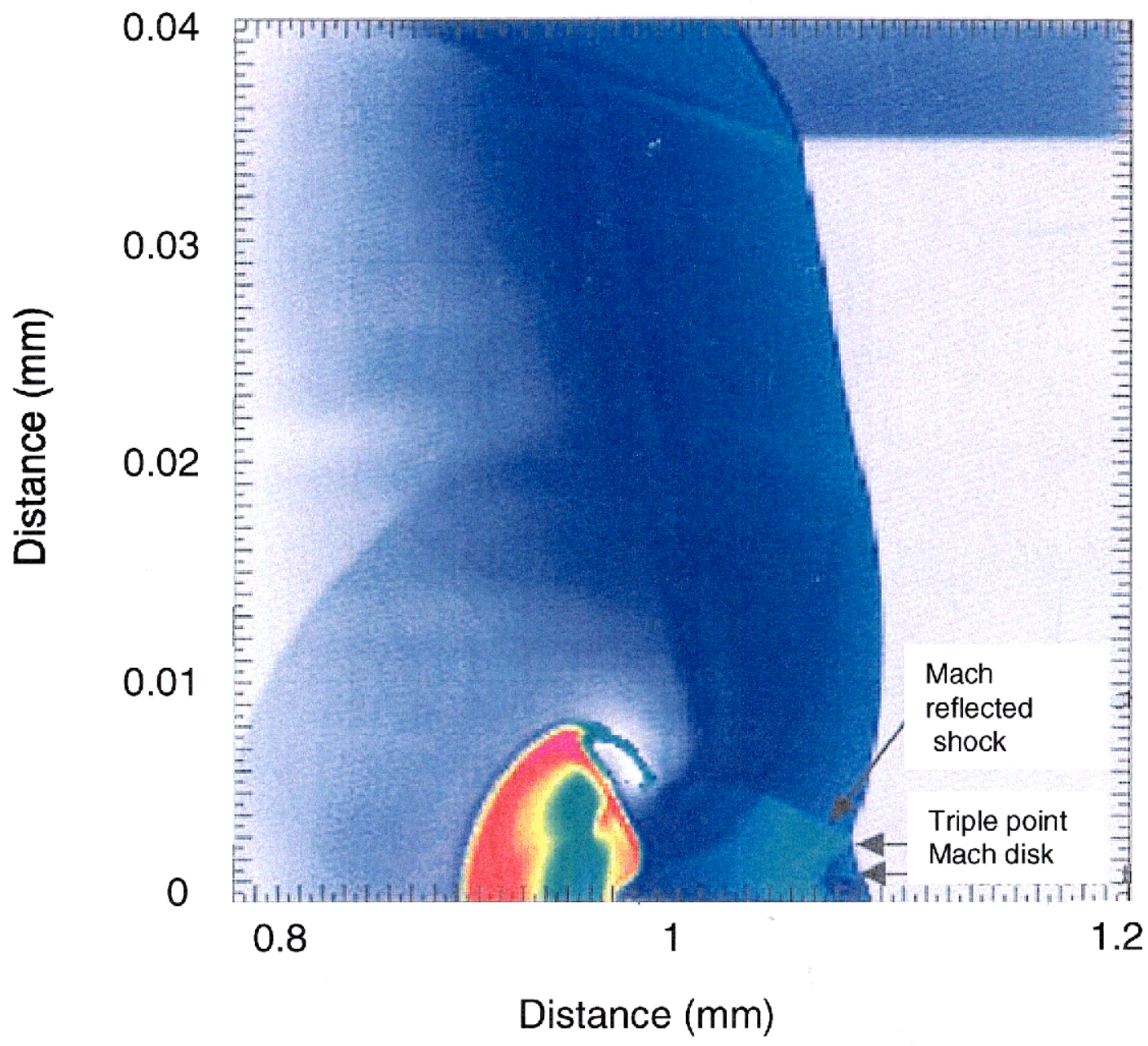

Figure 6 


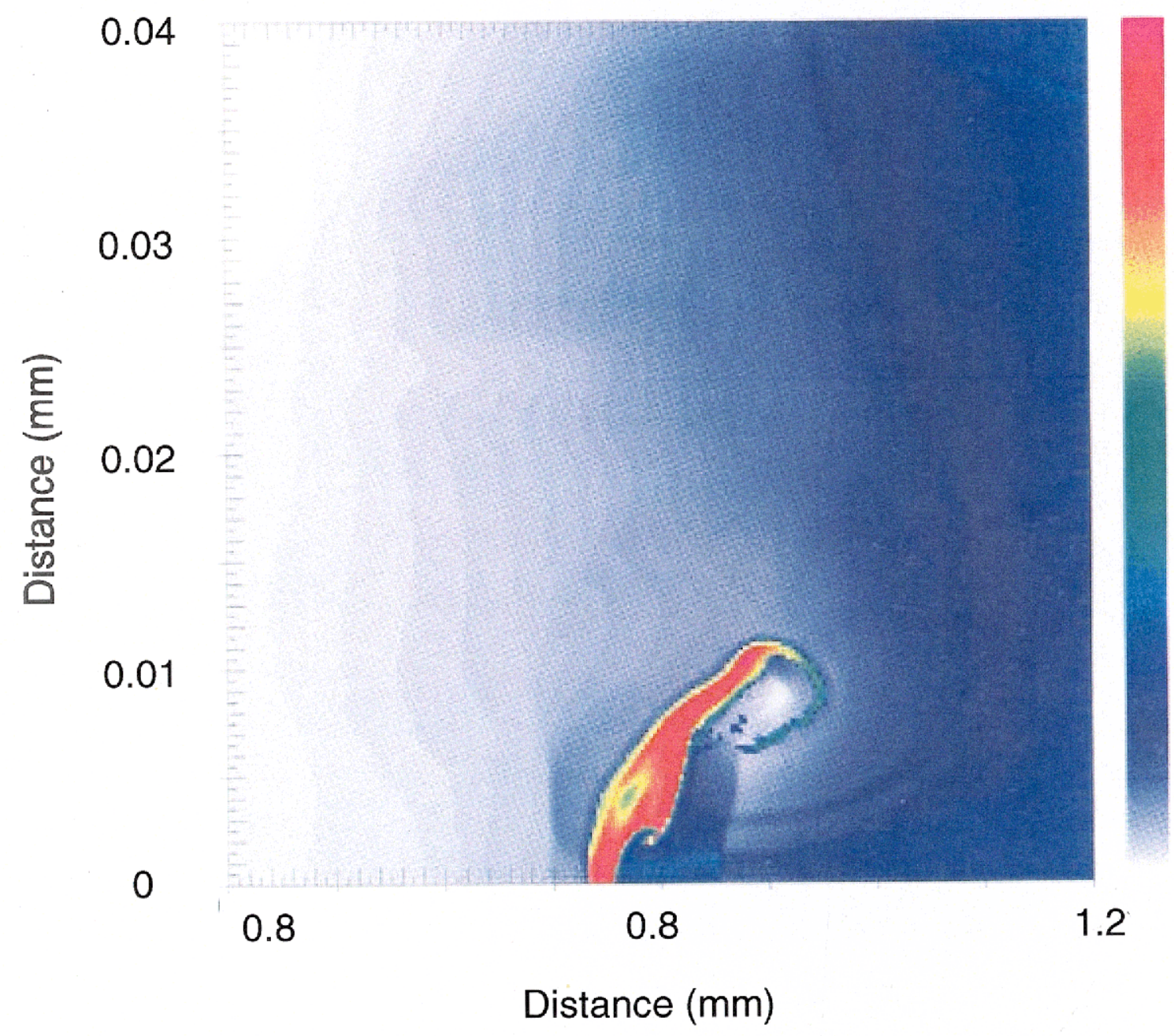

Figure 7 


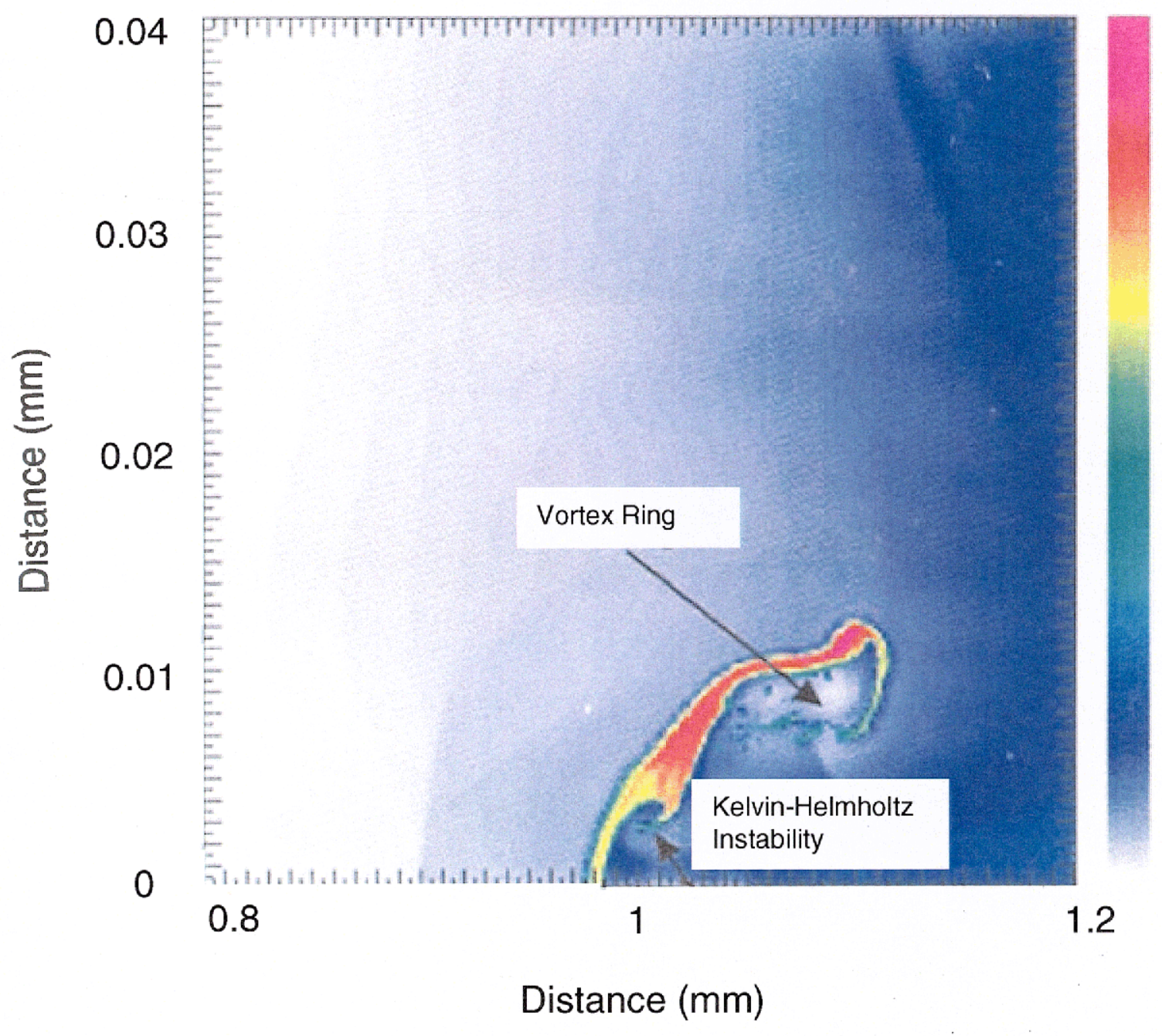

Figure 8 


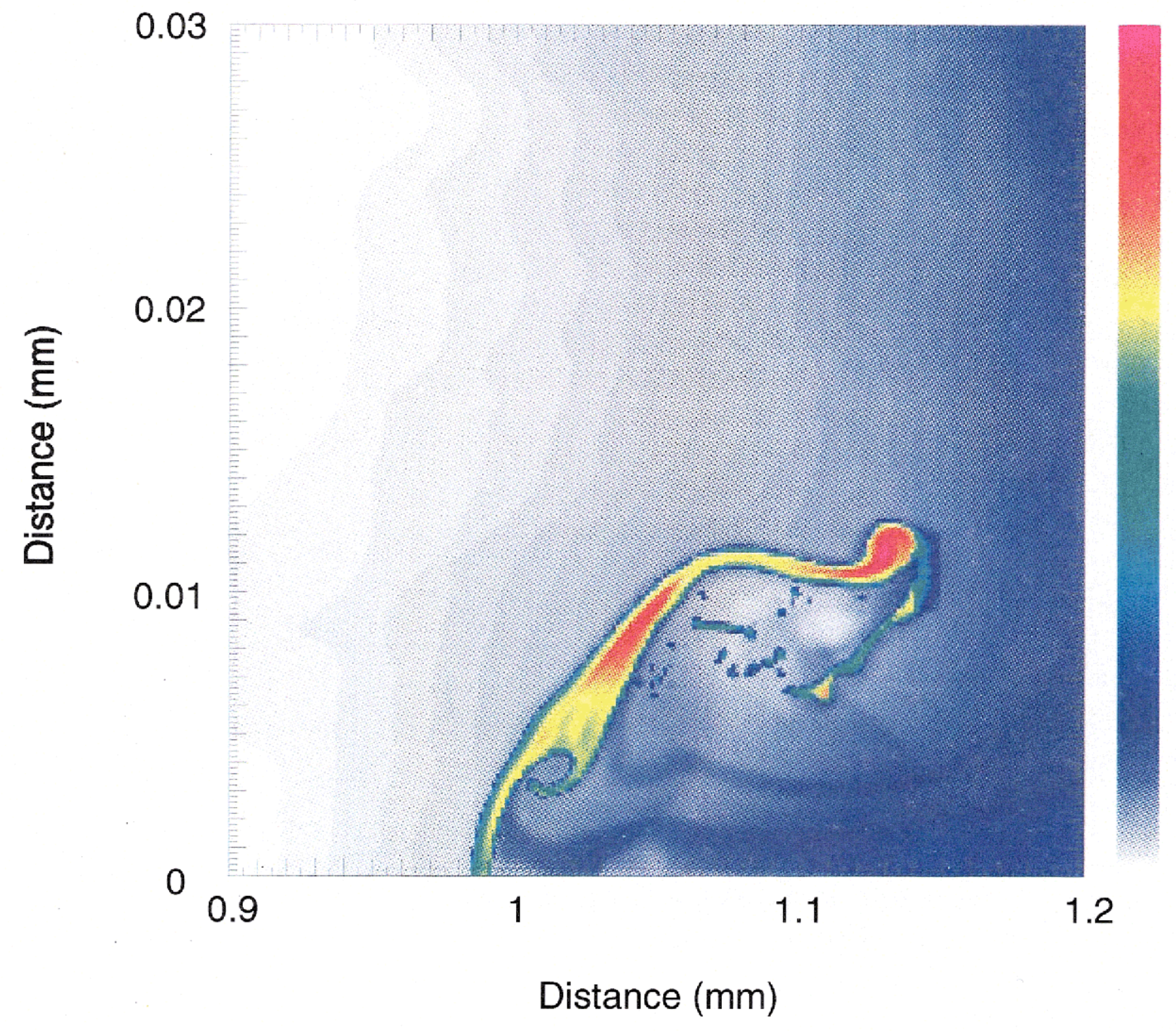

Figure 9 


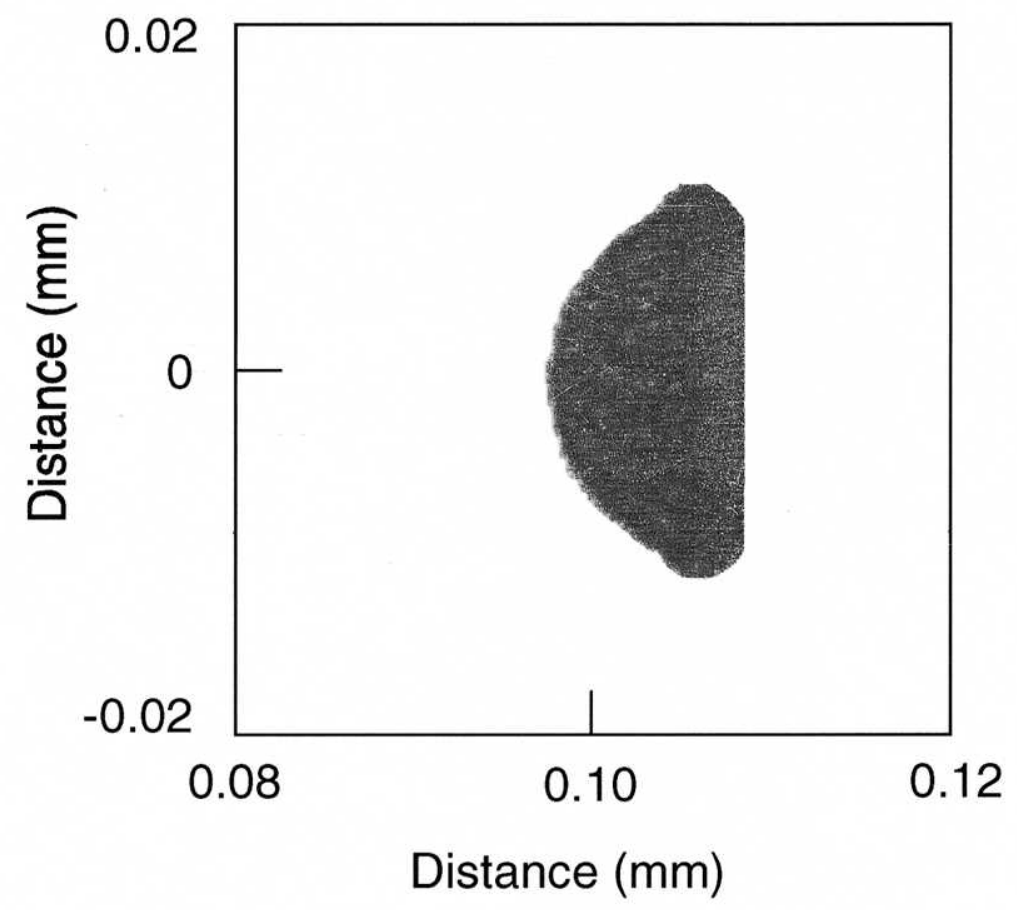

Figure 10 


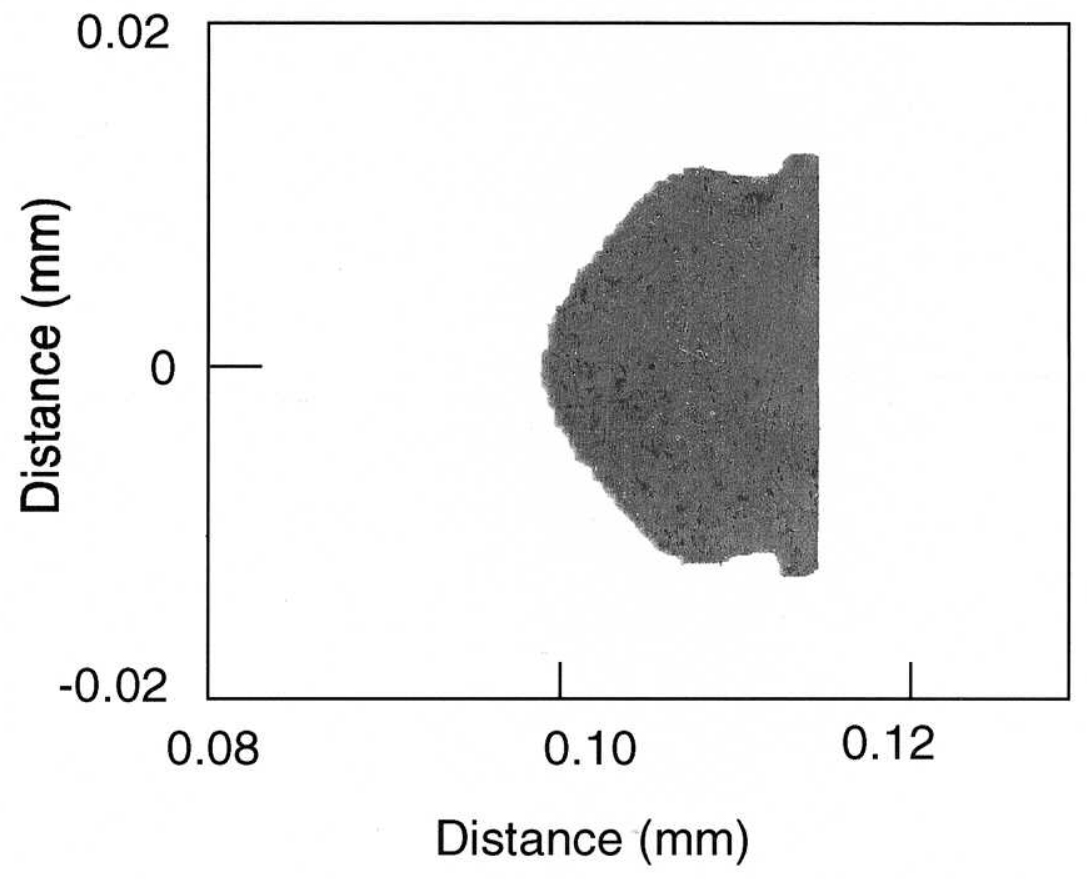

Figure 11 


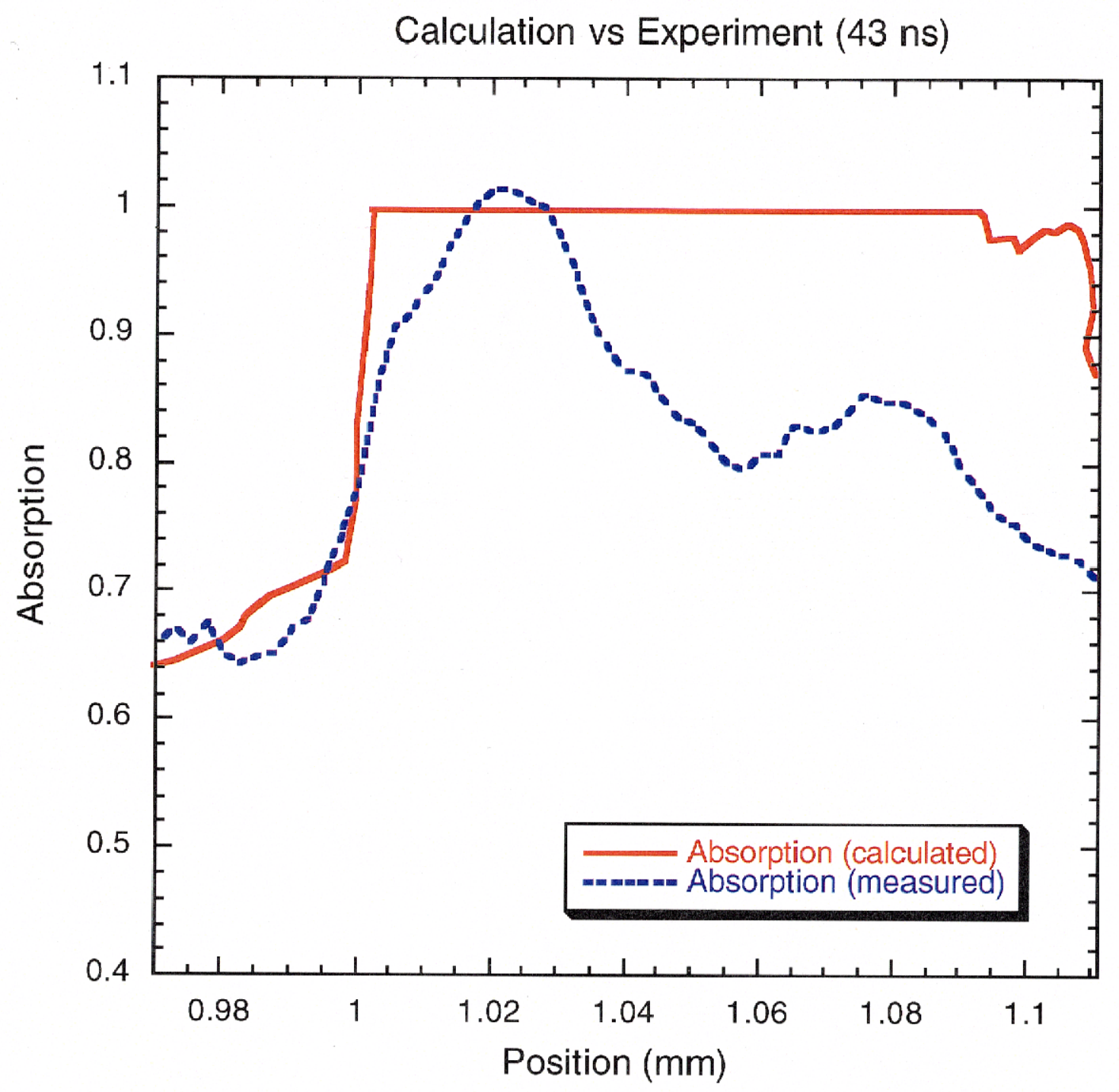

Figure 12 


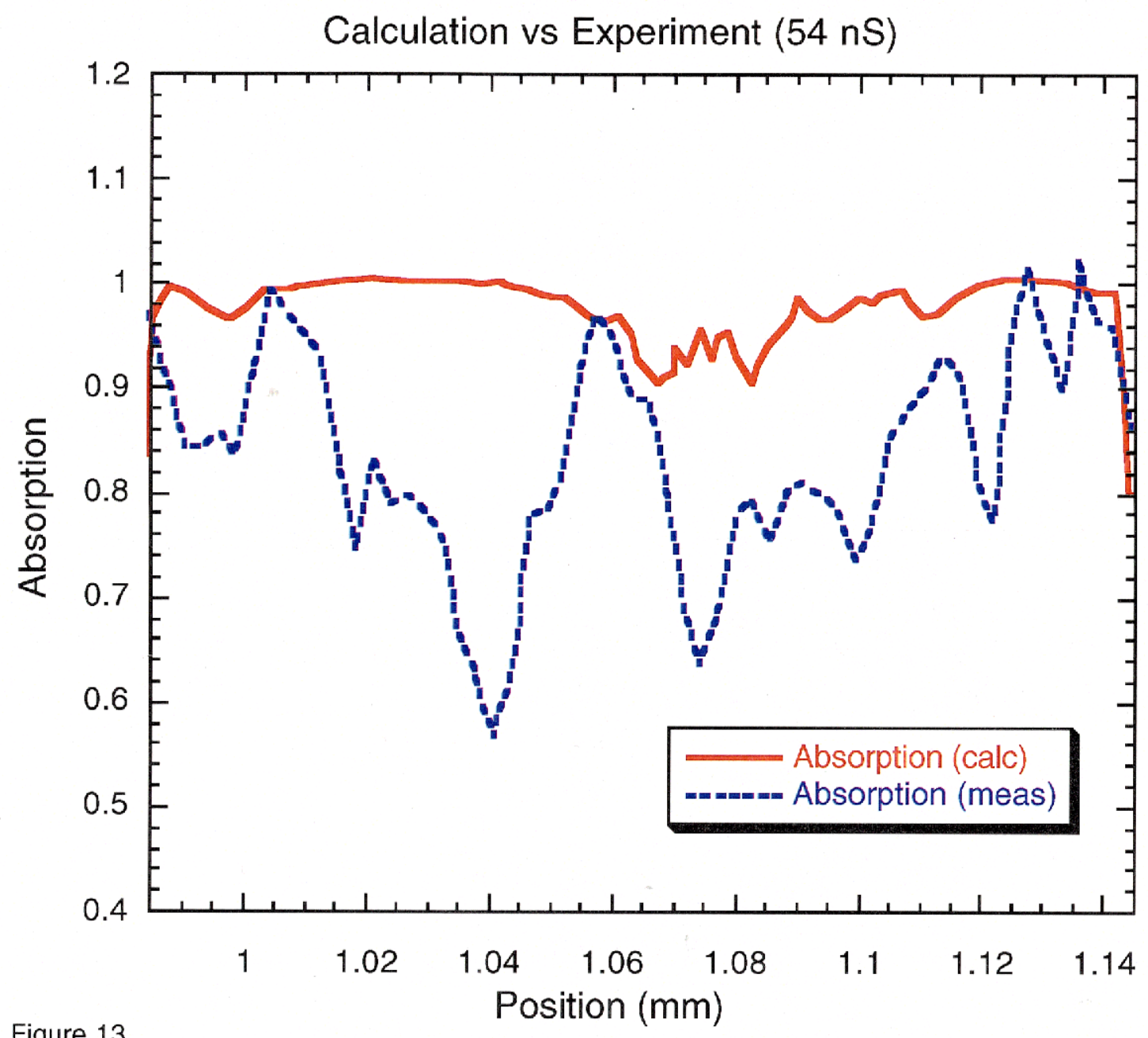

Figure 13 


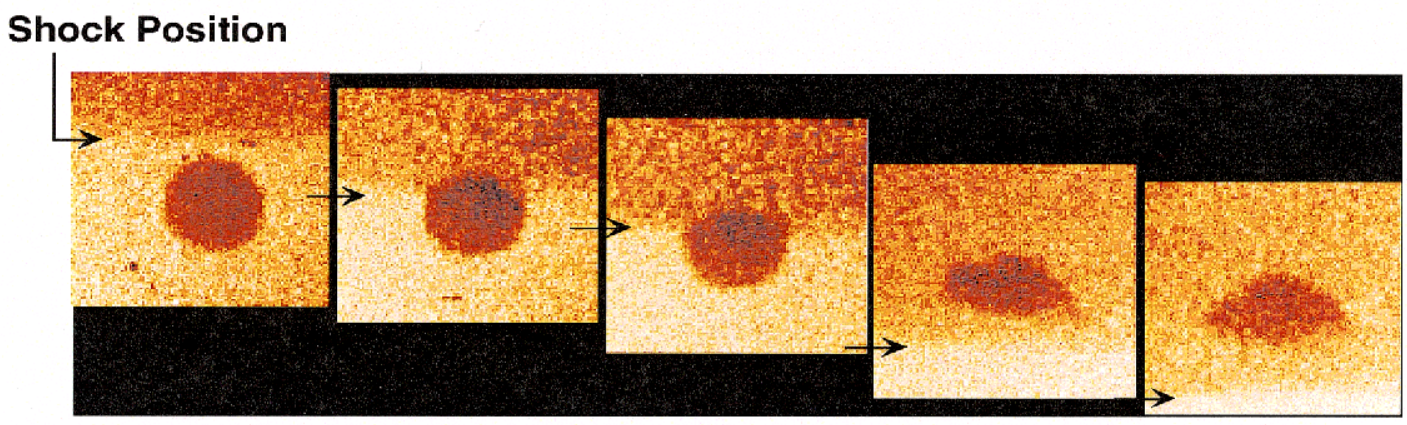

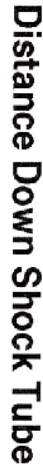

Figure 14 

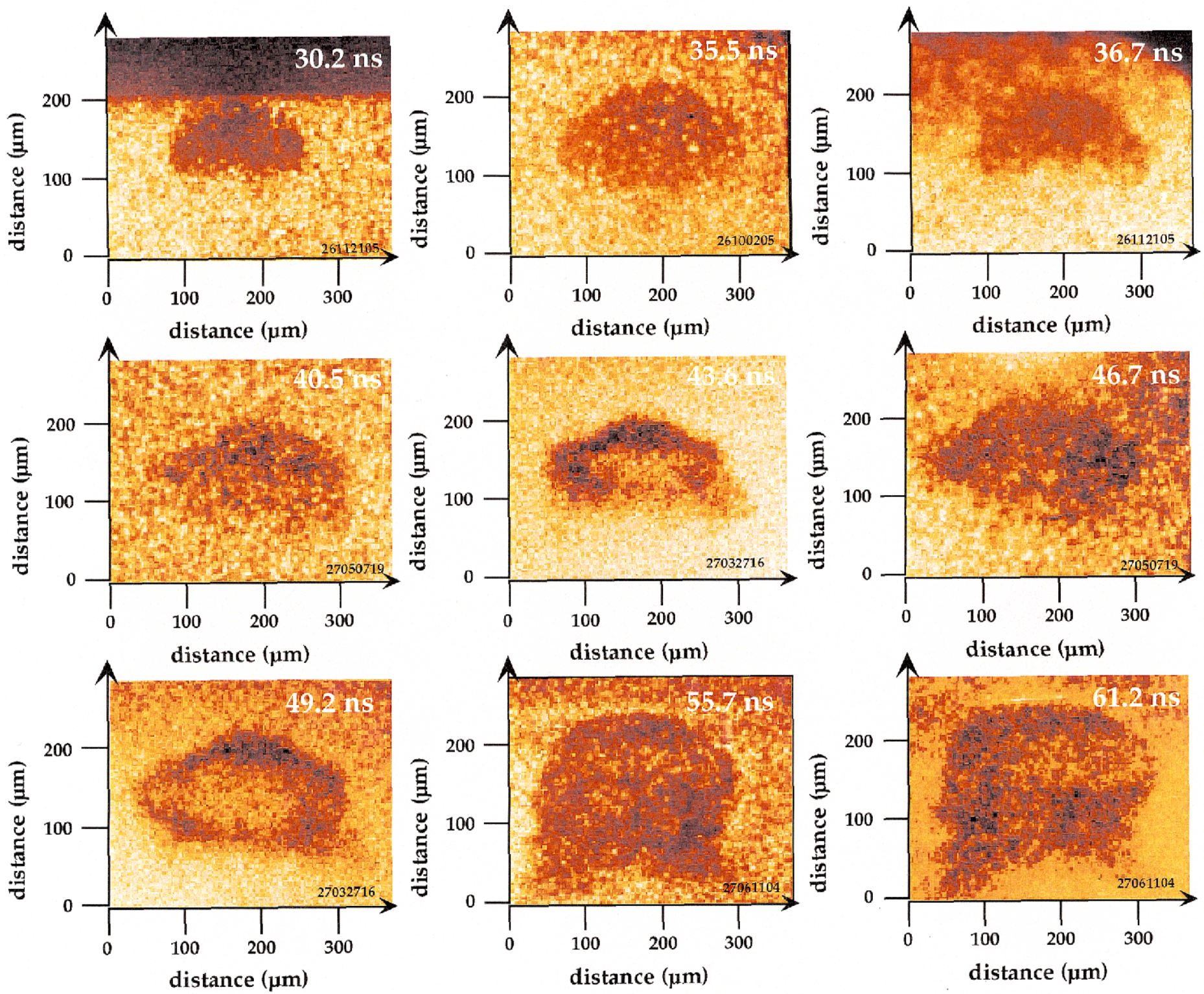

Figure 15 


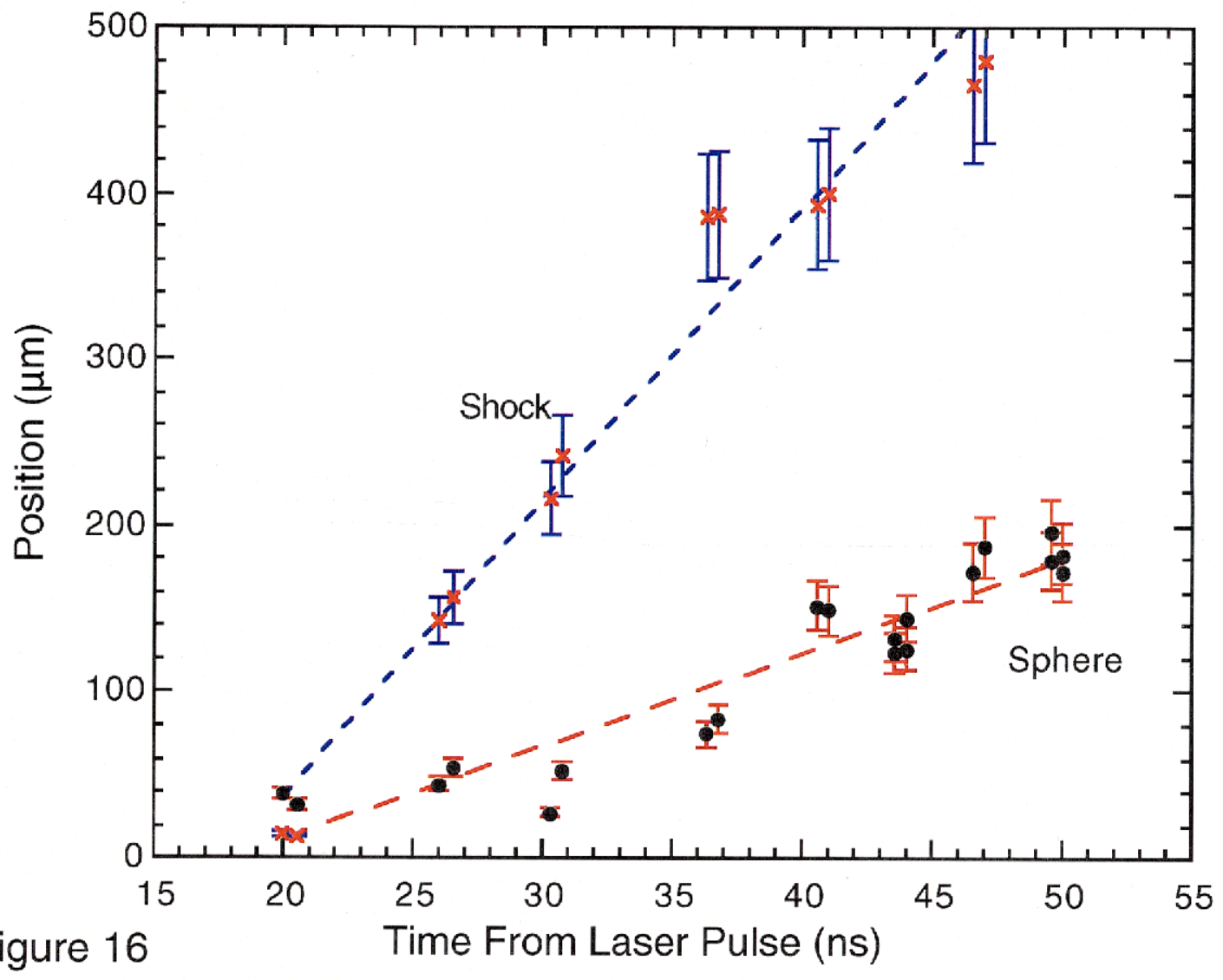




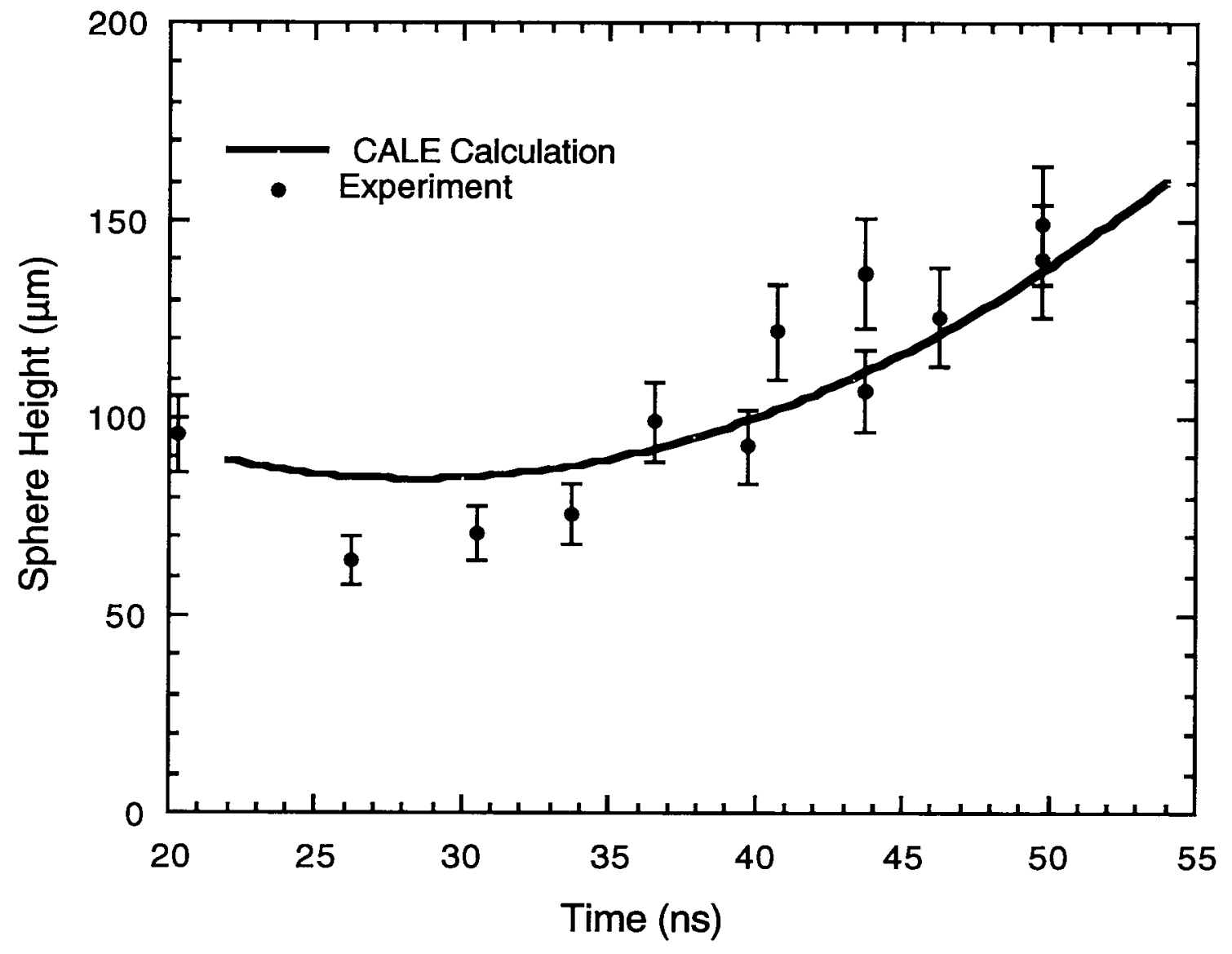

Figure 17 


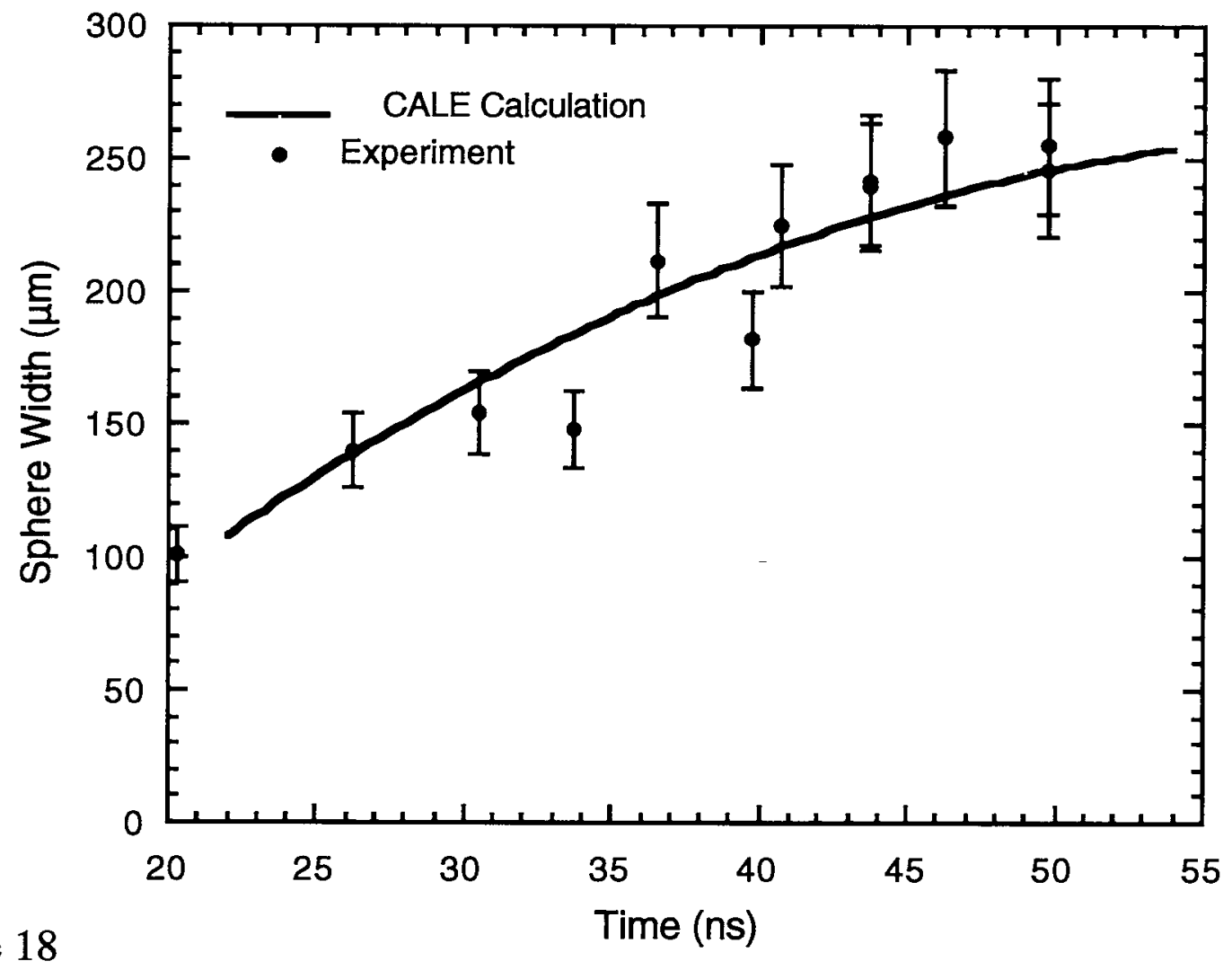

Figure 18 


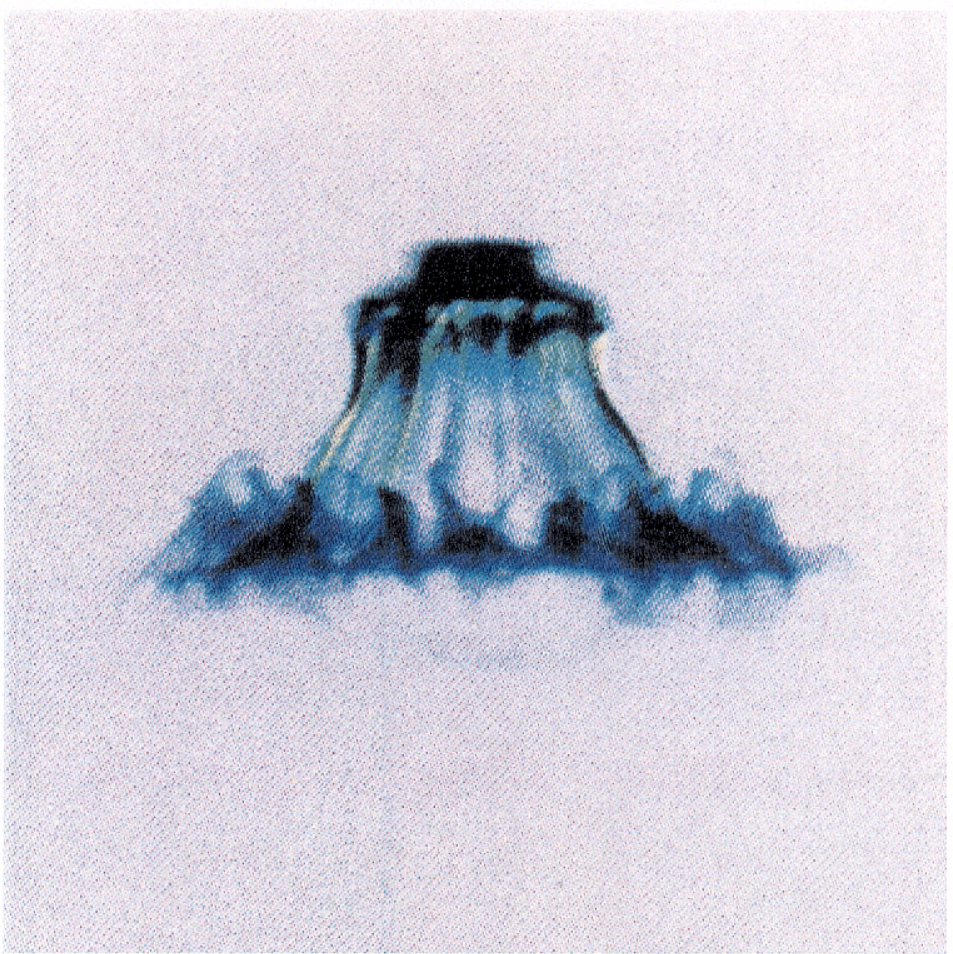

Figure 19 